\title{
Improvement of hierarchical matrices for 3D elastodynamic problems with a complex wavenumber
}

\author{
Laura Bagur · Stéphanie Chaillat • Patrick Ciarlet Jr.
}

the date of receipt and acceptance should be inserted later

\begin{abstract}
It is well known in the literature that standard hierarchical matrix ( $\mathcal{H}$-matrix) based methods, although very efficient for asymptotically smooth kernels, are not optimal for oscillatory kernels. In a previous paper, we have shown that the method should nevertheless be used in the mechanical engineering community due to its still important data-compression rate and its straightforward implementation compared to $\mathcal{H}^{2}$-matrix, or directional, approaches.

Since in practice, not all materials are purely elastic it is important to be able to consider visco-elastic cases. In this context, we study the effect of the introduction of a complex wavenumber on the accuracy and efficiency of $\mathcal{H}$-matrix based fast methods for solving dense linear systems arising from the discretization of the elastodynamic (and Helmholtz) Green's tensors. Interestingly, such configurations are also encountered in the context of the solution of transient purely elastic problems with the convolution quadrature method. Relying on the theory proposed in [12] for $\mathcal{H}^{2}$-matrices for Helmholtz problems, we study the influence of the introduction of damping on the data compression rate of standard $\mathcal{H}$-matrices. We propose an improvement of $\mathcal{H}$-matrix based fast methods for this kind of configuration. This work is complementary to the recent work [12]. Here, in addition to addressing another physical problem, we consider standard $\mathcal{H}$-matrices, derive a simple criterion to introduce additional compression and we perform extensive numerical experiments.
\end{abstract}

Keywords Hierachical matrices · Convolution Quadrature Method · Boundary Element Method · (Visco)-Elastodynamics

\section{Introduction}

The 3D linear isotropic elastodynamic equation for the displacement field $\boldsymbol{u}$ (also called Navier equation) is given by

$$
\operatorname{div} \sigma(\boldsymbol{u})+\rho \omega^{2} \boldsymbol{u}=0
$$

where $\omega>0$ is the circular frequency. It is supplemented with appropriate boundary conditions which contain the data. The stress and strain tensors are respectively given by $\sigma(\boldsymbol{u})=\lambda(\operatorname{div} \boldsymbol{u}) \mathbf{I}_{3}+2 \mu \varepsilon(\boldsymbol{u})$ and $\varepsilon(\boldsymbol{u})=\frac{1}{2}\left([\boldsymbol{\nabla} \boldsymbol{u}]+[\nabla \boldsymbol{u}]^{\top}\right)$, where $\mathbf{I}_{3}$ is the 3-by-3 identity matrix and $[\nabla \boldsymbol{u}]$ is the 3-by-3 matrix whose $\beta$-th column is the gradient of the $\beta$-th component of $\boldsymbol{u}$ for $1 \leq \beta \leq 3, \mu$ and $\lambda$ are the Lamé parameters and $\rho$ is the density.

Denoting $k_{p}^{2}=\rho \omega^{2}(\lambda+2 \mu)^{-1}$ and $k_{s}^{2}=\rho \omega^{2} \mu^{-1}$ the so-called P and S wavenumbers, the Green's tensor of the Navier equation is a 3-by-3 matrix-valued function expressed by

$$
\mathbf{U}_{\omega}(\boldsymbol{x}, \boldsymbol{y})=\frac{1}{\rho \omega^{2}}\left(\boldsymbol{c u r l} \boldsymbol{c u r l} \boldsymbol{l}_{\boldsymbol{x}}\left[\frac{e^{i k_{s}|\boldsymbol{x}-\boldsymbol{y}|}}{4 \pi|\boldsymbol{x}-\boldsymbol{y}|} \mathbf{I}_{3}\right]-\boldsymbol{\nabla}_{\boldsymbol{x}} \operatorname{div}_{\boldsymbol{x}}\left[\frac{e^{i k_{p}|\boldsymbol{x}-\boldsymbol{y}|}}{4 \pi|\boldsymbol{x}-\boldsymbol{y}|} \mathbf{I}_{3}\right]\right)
$$

where the index $\boldsymbol{x}$ means that differentiation is carried out with respect to $\boldsymbol{x}$ and $\operatorname{div}_{\boldsymbol{x}} \mathbb{A}$ corresponds to the application of the divergence along each row of $\mathbb{A}$. One may use this tensor to represent the solution of (1). Alternately, one may use the tensor $\mathbf{T}_{\omega}(\boldsymbol{x}, \boldsymbol{y})$, which is obtained by applying the traction operator

$$
\boldsymbol{T}=2 \mu \frac{\partial}{\partial \boldsymbol{n}}+\lambda \boldsymbol{n} \operatorname{div}+\mu \boldsymbol{n} \times \boldsymbol{c u r l}
$$

to each column of $\mathbf{U}_{\omega}(\boldsymbol{x}, \boldsymbol{y}): \mathbf{T}_{\omega}(\boldsymbol{x}, \boldsymbol{y})=\left[\boldsymbol{T}_{\boldsymbol{y}} \mathbf{U}_{\omega}(\boldsymbol{x}, \boldsymbol{y})\right]$ where the index $\boldsymbol{y}$ means that differentiation is carried out with respect to $\boldsymbol{y}$.

Laboratoire POEMS (CNRS-INRIA-ENSTA Paris), ENSTA Paris, Institut Polytechnique de Paris, 828 Bd des Maréchaux, Palaiseau, 91120 , France E-mail: laura.bagur@ensta-paris.fr·stephanie.chaillat@ensta-paris.fr·patrick.ciarlet@ensta-paris.fr 
We consider the fast solution of dense linear systems of the form

$$
\mathbb{A} \mathbf{p}=\mathbf{b}, \quad \mathbb{A} \in \mathbb{C}^{3 N_{c} \times 3 N_{c}}
$$

where $\mathbb{A}$ is the matrix corresponding to the discretization of the 3-by-3 Green's tensors $\mathbf{U}_{\omega}\left(\boldsymbol{x}_{i}, \boldsymbol{y}_{j}\right)$ or $\mathbf{T}_{\omega}\left(\boldsymbol{x}_{i}, \boldsymbol{y}_{j}\right)$ for two clouds of $N_{c}$ points $\left(\boldsymbol{x}_{i}\right)_{1 \leq i \leq N_{c}}$ and $\left(\boldsymbol{y}_{j}\right)_{1 \leq j \leq N_{c}}$. Here $\mathbf{p}$ is the unknown vector approximating the solution at $\left(\boldsymbol{x}_{i}\right)_{1 \leq i \leq N_{c}}$ and $\mathbf{b}$ is a given right hand side that depends on the data. Such dense systems are encountered for example in the context of the Boundary Element Method (with a straightforward derivation for piecewise constant interpolation of the unknown field) [5, 32].

If no compression or acceleration technique is used, the storage of such a system is of the order $O\left(N_{c}^{2}\right)$, the iterative solution (e.g. with GMRES) is $O\left(N_{\text {iter }} N_{c}^{2}\right)$ where $N_{\text {iter }}$ is the number of iterations, while the direct solution (e.g. via LU factorizations) is $O\left(N_{c}^{3}\right)$. In the last decades, different approaches have been proposed to speed up the solution of dense systems. The most well-known method is probably the fast multipole method (FMM) proposed by Greengard and Rokhlin [20] which enables a fast evaluation of the matrix-vector products. We recall that the matrix-vector product is the crucial tool in the context of an iterative solution. Initially developed for N-body simulations, the FMM has then been extended to oscillatory kernels [19,15]. The method is now widely used in many application fields and has shown its capabilities in the context of mechanical engineering problems solved with the BEM [13,24,36].

An alternative approach designed for dense systems is based on the concept of hierarchical matrices ( $\mathcal{H}$-matrices) [2]. The principle of $\mathcal{H}$-matrices is to partition the initial dense linear system, and then approximate it into a data-sparse one, by finding sub-blocks in the matrix that can be accurately estimated by low-rank matrices. In other terms, one further approximates the matrix $\mathbb{A}$ from $(4)$. The efficiency of hierarchical matrices relies on the possibility to approximate, under certain conditions, the underlying kernel function by low-rank matrices. The approach has been shown to be very efficient for asymptotically smooth kernels (e.g. Laplace kernel). On the other hand, oscillatory kernels such as the Helmholtz or elastodynamic kernels, are not asymptotically smooth. In these cases, the method is not optimal [1]. To avoid the increase of the rank for high-frequency problems, $\mathcal{H}^{2}$-matrix, or directional, methods have been proposed [6,7]. $\mathcal{H}^{2}$-matrices are a specialization of hierarchical matrices. It is a multigrid-like version of $\mathcal{H}$-matrices that enables more compression, by factorizing some basis functions of the approximate separable expansion.

Since the implementation of $\mathcal{H}^{2}$-matrix methods is much more involved than the one of the standard $\mathcal{H}$-matrix, in [14] we have studied the frequency-range within which the $\mathcal{H}$-matrices are efficient for elastodynamic problems and what can be expected of such an approach to solve problems encountered in mechanical engineering. We have shown that even though the method is not optimal (in the sense that more efficient approaches can be proposed at the cost of a much more complex implementation effort), an efficient solver is easily developed. The capabilities of the method have been illustrated on numerical examples using the Boundary Element Method.

In practice, not all materials are purely elastic and it is thus important to be able to consider visco-elastic cases. In this context, we study the effect of the introduction of a complex wavenumber on the accuracy and efficiency of hierarchical matrix $(\mathcal{H}$-matrix) based fast methods for solving dense linear systems arising from the discretization of the elastodynamic Green's tensors. Interestingly, such configurations are also encountered in the context of the solution of transient purely elastic problems with the convolution quadrature method. Relying on the theory proposed in [12] for $\mathcal{H}^{2}$-matrices for Helmholtz problems, we study the influence of the introduction of damping on the data compression rate of standard $\mathcal{H}$-matrices. We propose an improvement of $\mathcal{H}$-matrix based fast methods for this kind of configuration. This work is complementary to the recent report [12]. Here, in addition to addressing another physical problem, we consider standard $\mathcal{H}$-matrices, derive an additional condition to obtain more compression and we perform extensive numerical experiments.

This paper is organized as follows. In Section 2, we recall the main algorithmic components of standard $\mathcal{H}$-matrices. Then in Section 3, we review existing and improved admissibility conditions for the case of complex wavenumbers. We discuss the similarities, differences and novelties compared to [12]. In Section 4, we perform extensive numerical tests to show the efficiency of a new admissibility condition, designed to improve the efficiency of standard $\mathcal{H}$-matrices in the case of complex wavenumbers. Section 5 is devoted to the discussion of some practical situations in which this improved approach will be useful. The paper ends with some conclusions and future works.

\section{Main components of $\mathcal{H}$-matrices}

Hierarchical matrices or $\mathcal{H}$-matrices have been introduced by Hackbusch [21] to compute a data-sparse representation of some special dense matrices (e.g., matrices resulting from the discretization of non-local operators). The principle of $\mathcal{H}$ matrices is (i) to partition the matrix into blocks and (ii) to perform low-rank approximations of the blocks of the matrix which are known a priori (by using an admissibility condition) to be accurately approximated by low-rank decompositions. With these two ingredients it is possible to define fast iterative and direct solvers for matrices having a hierarchical representation. Using low-rank representations, the memory requirements and costs of a matrix-vector product are reduced. In addition, using $\mathcal{H}$-matrix arithmetic it is possible to derive fast direct solvers. 
Clustering of the unknowns The key ingredient of hierarchical matrices is the recursive block subdivision. The first step, prior to the partition of the matrix, is thus a partitioning based on the geometry of the set of row and column indices of the matrix $\mathbb{A}$. The purpose is to perform a permutation of the indices in the matrix to reflect the physical distance and thus interaction between degrees of freedom. Consecutive indices should correspond to DOFs that interact at close range. For the sake of clarity, in this work $\mathbb{A}$ is defined by the same set of indices $I=\{1, \ldots, n\}$ for rows and columns. A binary tree $\mathcal{T}_{I}$ is used to drive the clustering. Each node of the tree defines a subset of indices $\sigma \subset I$ and each subset corresponds to a part in the partition of the domain. There exist different approaches to perform the subdivision [22]. We consider the simplest possible one : based on a geometric argument. For each node in the tree, we determine the box enclosing all the points in the cloud and subdivide it into two balanced boxes, along the largest dimension. The subdivision is stopped when a prescribed number of points per box $N_{\text {leaf }}$, is reached. The depth of the tree $\mathcal{T}_{I}$ is denoted by $L(I)$.

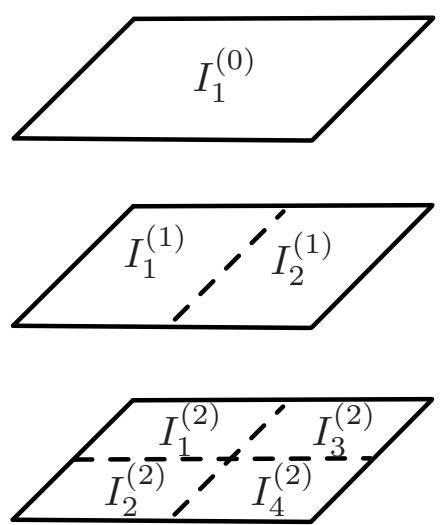

(a) Partition of the physical domain

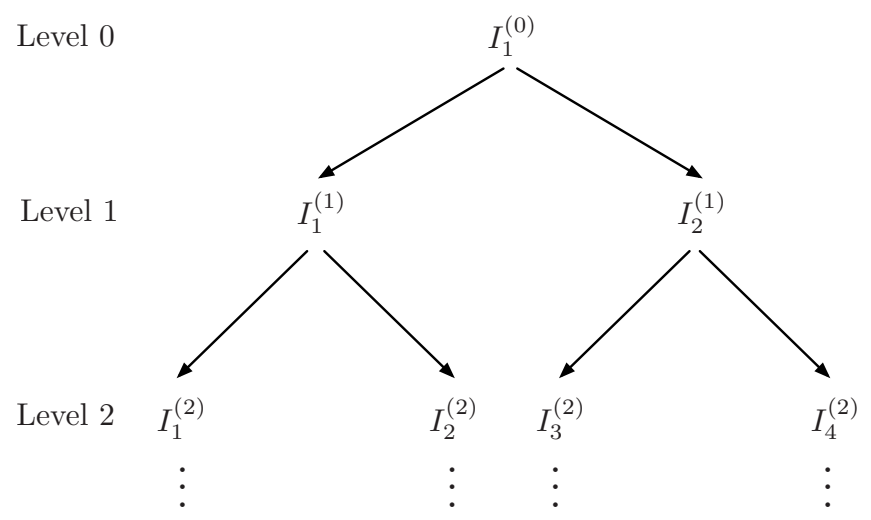

(b) Binary cluster tree $\mathcal{T}_{I}$

Fig. 1: Illustration of the clustering of the degrees of freedom: (a) partition of the degrees of freedom in the domain and (b) corresponding binary tree.

Subdivision of the matrix After the clustering of the unknowns is performed, a block cluster representation $\mathcal{T}_{I \times I}$ of the matrix $\mathbb{A}$ is defined by going through the cluster tree $\mathcal{T}_{I}$. Each node of $\mathcal{T}_{I \times I}$ contains a pair $(\sigma, \tau)$ of indices of $\mathcal{T}_{I}$ and defines a block of $\mathbb{A}$ (see Figure 2). This uniform partition defines a block structure of the matrix with a full pattern of $4^{L(I)-1}$ blocks, in particular every node of the tree at the leaf level is connected with all the other nodes at the leaf level (Figure 3 a). This partition is not optimal since some parts of the matrix $\mathbb{A}$ can accurately be approximated by a low-rank matrix at a higher level (i.e. for larger clusters). Such blocks are said to be admissible. A hierarchical representation $\mathcal{P} \subset \mathcal{T}_{I \times I}$ that uses the cluster tree $\mathcal{T}_{I}$ and the existence of admissible blocks is more appropriate. Starting from the initial matrix, each block is recursively subdivided until it is either admissible or the leaf level is reached. For complex 3D geometries, an admissibility condition based on the geometry and the interaction distance between points is used to determine a priori the admissible blocks. For more details on the construction of the block cluster tree, we refer the interested reader to [9]. The partition $\mathcal{P}$ is subdivided into two subsets $\mathcal{P}^{\text {ad }}$ and $\mathcal{P}^{\text {non-ad }}$ reflecting the possibility for a block $\tau \times \sigma$ to be either admissible, i.e., $\tau \times \sigma \in \mathcal{P}^{\text {ad }}$; or non-admissible, i.e., $\tau \times \sigma \in \mathcal{P}^{\text {non-ad }}$. It is clear that $\mathcal{P}=\mathcal{P}^{\text {ad }} \cup \mathcal{P}^{\text {non-ad }}$. To sum up, the blocks of the partition can be of three types: at the leaf level a block can be either an admissible block or a non-admissible block, at a non-leaf level a block can be either an admissible block or an $\mathcal{H}$-matrix (i.e, a block that will be subsequently hierarchically subdivided).

Performing low-rank approximations. Once the admissible blocks are determined, an accurate rank-revealing algorithm is applied to determine low-rank approximations. We recall that the numerical rank of a matrix $\mathbb{A}$ is

$$
r(\varepsilon):=\min \left\{r \quad \mid \quad\left\|\mathbb{A}-\mathbb{A}_{r}\right\| \leq \varepsilon\|\mathbb{A}\|\right\}
$$

where $\mathbb{A}_{r}$ defines the singular value decomposition (SVD) of $\mathbb{A}$ keeping only the $r$ largest singular values and $\varepsilon>0$ is a given parameter. Such an algorithm must be accurate (i.e., its result, the computed numerical rank, must be as small as possible) to avoid unnecessary computational costs. The truncated Singular Value Decomposition (SVD) [16] gives the best low-rank approximation (Eckart-Young theorem) for unitary invariant norms (e.g., Frobenius or spectral norm). Thus it produces an approximation with the smallest possible numerical rank for a given prescribed accuracy. But the computation of the SVD is expensive, i.e., in the order of $O\left(\max (m, n) \times \min (m, n)^{2}\right)$ for an $m \times n$ matrix, and in addition it requires the computation 


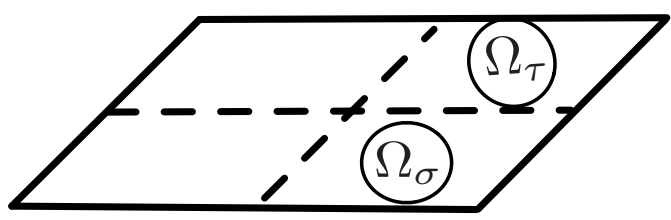

(a)

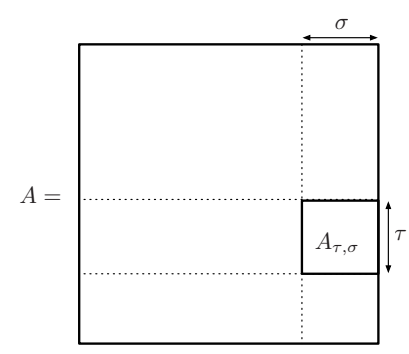

(b)

Fig. 2: Illustration of the construction of the block cluster tree: (a) Clustering of the unknowns on the geometry and (b) corresponding block clustering in the matrix.

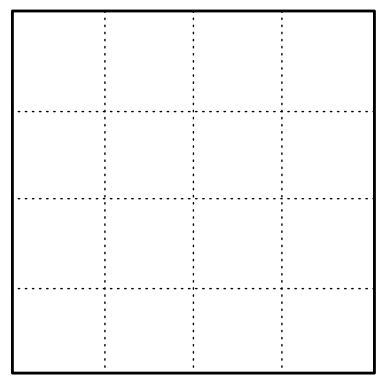

(a)

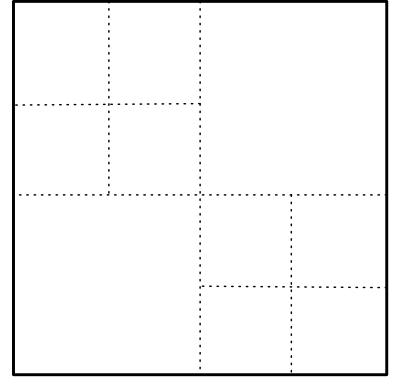

(b)

Fig. 3: (a) Block cluster representation $\mathcal{T}_{I \times I}$ for the illustrative example (full structure); (b) Hierarchical partition $\mathcal{P} \subset \mathcal{T}_{I \times I}$ of the same matrix based on the admissibility condition (sparse structure).

of all the entries of $\mathbb{A}$. In the context of the $\mathcal{H}$-matrices, the use of the SVD would induce the undesired need to assemble the complete matrix.

The adaptive cross approximation (ACA) [3, 4] offers an interesting alternative to the SVD since it produces a quasioptimal low-rank approximation without requiring the assembly of the complete matrix. The starting point of the ACA is that every matrix of rank $r$ is the sum of $r$ matrices of rank 1. The ACA is thus a greedy algorithm that improves the accuracy of the approximation by adding iteratively rank-1 matrices. There are various ACAs that differ by the choice of the best pivot at each iteration. The simplest approach is the so-called fully-pivoted ACA and it consists in choosing the pivot as the largest entry in the residual. But similarly to the SVD, it requires the computation of all the entries of $\mathbb{A}$ to compute the pivot indices. It is not an interesting option for the construction of $\mathcal{H}$-matrices. The partially-pivoted ACA proposes an alternative approach to choose the pivot avoiding the assembly of the complete matrix. The idea is to maximize alternately the residual for only one of the two indices and to keep the other one fixed. With this strategy, only one row and one column is assembled at each iteration. The complexity of the partially-pivoted ACA is reduced to $O\left(r_{\mathrm{ACA}}^{2}(m+n)\right)$, where $r_{\mathrm{ACA}}$ is the achieved rank. This is the approach used in this work but the results presented do not depend on this choice. Other approaches such as fast multipole expansions [30,20], panel clustering [31,23], quadrature formulas [8] or interpolations [29] could be used. The advantages of the ACA are to be purely algebraic and easy to implement.

\section{Existing admissibility conditions and improvements for the case of complex wavenumbers}

The admissibility condition enables to distinguish blocks which are known a priori to be accurately approximated by a lowrank approximation. The standard admissibility condition for $\mathcal{H}$-matrices, optimal for asymptotically smooth kernels and efficient for oscillatory kernels is given by

$$
\text { the block } X \times Y \text { is admissible if } \min (\operatorname{diam}(X), \operatorname{diam}(Y)) \leq \eta \operatorname{dist}(X, Y)
$$

with diam $(X)$, diameter of the cluster $X$ (in practice the diameter of the bounding box), and dist $(X, Y)$, the distance between the clusters $X$ and $Y$ (the distance between the bounding boxes in practice) - See Fig. 4 . 


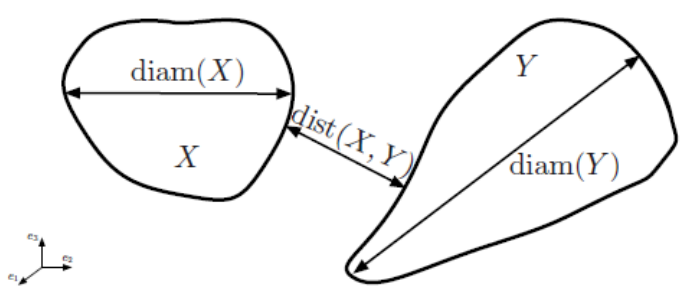

(a)

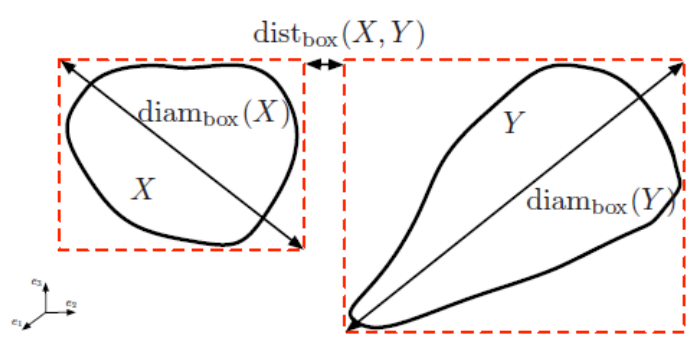

(b)

Fig. 4: Efficient implementation of the admissibility condition: (a) theoretical condition and (b) implemented condition.

Sparsification condition for damped kernels. In the context of oscillatory kernels with complex wavenumbers, we know that $\frac{\exp \left(i k_{\beta} r\right)}{r}$ is the dominant term of the $3 \mathrm{D}$ elastodynamic kernel function. A first issue is thus to determine when the negative exponential term introduced in the kernel, due to $\operatorname{Im}\left(k_{\beta}\right)>0$ (with $\beta=s$ or $p$ ), dominates the oscillatory behaviour. For a given wavenumber $k_{\beta} \in \mathbb{C}$ with $\operatorname{Im}\left(k_{\beta}\right)>0$, the question thus reduces to find the smallest value $r_{\text {lim }}>0$ such that :

$$
\forall r \geq r_{\text {lim }},\left|\frac{\exp \left(i k_{\beta} r\right)}{r}\right| \leqslant \varepsilon_{\text {decay }} \times\left|\frac{\exp \left(i k_{\beta} r_{\text {min }}\right)}{r_{\min }}\right|
$$

In (7), there are two parameters:

- $r_{\text {min }}$, which is related to the mesh. It can be defined for example as the minimal distance between two nodes of the boundary element mesh (importantly it is thus also related to the minimal distance between two clusters).

$-\varepsilon_{\text {decay }}$, which is the threshold parameter of the sparsification.

If we can define for a given wavenumber such an $r_{\text {lim }}$, we know that some blocks of the matrix will be full of zeros, or at least all their entries are sufficiently small so that the approximation can be chosen as a 0-rank approximation, i.e., it is also full of zeros. The aim of this Section is thus to determine if we can complement the standard admissibility condition 6 to take into account these sparse blocks.

In the discussion below, the wavenumber is denoted by $k \in \mathbb{C}$ with $\operatorname{Im}(k)>0$. In [12], sparsification of oscillatory kernels with damping is also considered. One adjustable parameter is the order $\tilde{m}$ of the polynomials required to approximate the oscillatory kernel on a given admissible block $X \times Y$. Precisely in [12, definition (3.9)] the order of approximation $\tilde{m}$ is chosen so that the Chebyshev interpolation of the oscillatory kernel on the given admissible block $X \times Y$ achieves the requested approximation error. It is noted that if the resulting order is strictly lower than $0(\tilde{m}=-1)$, then the entries are sufficiently small so that the approximation is made of zeros. In this particular case the approximation boils down to a 0-rank approximation. Interestingly, a rigorous mathematical analysis is carried out, and the order of approximation $\tilde{m}$ on the given admissible block $X \times Y$ is chosen so that (see [12, bottom of page 11]):

$$
C_{0} \rho_{0}^{-\tilde{m}}\left|\frac{\exp (i \sigma k r)}{r}\right| \leq \varepsilon_{\text {error }}
$$

where $\varepsilon_{\text {error }}>0$ is the target approximation error, while $\sigma, C_{0}>0$, and $\rho_{0}>1$ are given values (see again [12, $\left.\S 4\right]$ ). So, up to some scaling factor, we note that condition (8) with $\tilde{m}=-1$ is completely similar to the proposed condition (7). Interestingly, even though empirical (7) is based on a sound mathematical analysis thanks to the equivalent condition (8) that is proposed and analyzed in [12]. Now, there remains to take into account those blocks that fulfil condition (7). We propose a heuristic condition, see (9] below. The major difference with [12] is that we do not limit the search to admissible blocks.

The first important remark concerns admissible blocks that fulfil condition (6) when an algebraic approach is used to perform the low rank approximation (which is another difference with [12]) . A priori, the numerical rank of a block full of zeros is 0 , so the ACA will automatically perform a low rank approximation with a rank equal to 0 . And if all the admissible blocks are blocks full of zeros then the maximal numerical rank of admissible blocks $r_{A C A}^{\max }$ is equal to 0 .

Second, one has to check whether some additional gains can be obtained on the storage of the non-admissible blocks, when they are made of entries with small/negligible values. This is the main originality of our new admissibility condition. In relation with the definition (7) of $r_{l i m}$, we add a condition based on the distance between clusters of non-admissible blocks. When $r_{A C A}^{\max }=0$, we propose the following simple admissibility condition to take into account potential non-admissible blocks full of zeros:

$$
\text { If } r_{A C A}^{\max }=0 \text {, a non-admissible block } X \times Y \text { is replaced by a matrix of zeros if } \operatorname{dist}(X, Y) \geq r_{l i m} \text {. }
$$


The aim of the additional admissibility condition $(9)$ is to further reduce storage requirements by approximating a large number of non-admissible blocks by matrices full of zeros. Note that it is tested only if all admissible blocks can already be approximated by matrices full of zeros, i.e., if $r_{A C A}^{\max }=0$. This is a conditional test that may, or may not, be implemented, in the limit of $\varepsilon_{\text {decay }}$ tending to $0^{+}$. Conditionality is expected to depend heavily on the damping.

Sparsification condition for oscillatory kernels. For an oscillatory kernel, possibly with damping, a well-known issue is to take into account its behavior depending on the direction. In the literature, this corresponds to the so-called $\mathcal{H}^{2}$-matrix, or directional, methods. We refer again to [12, definition 3.2], where an admissibility condition is proposed in the context of directional $\mathcal{H}^{2}$-matrices for Helmholtz problems with complex frequencies. For a wavenumber $k \in \mathbb{C}$ with $\operatorname{Im}(k)>0$, and given $\boldsymbol{\eta}=\left(\eta_{i}\right)_{i=1}^{3} \in \mathbb{R}_{>0}^{3}$, the admissibility condition writes: a pair of clusters $X, Y \in \mathcal{T}_{I}$ and a direction $c \in \mathbb{S}_{2}$ (unit sphere in $\mathbb{R}^{3}$ ) are said to be $\boldsymbol{\eta}$-admissible if they satisfy the following three conditions:

$$
\begin{aligned}
|R e(k)||| \frac{M_{X}-M_{Y}}{\operatorname{dist}\left(M_{X}, M_{Y}\right)}-c|| & \leqslant \frac{\eta_{1}}{\max \left\{\operatorname{diam}^{2}(X), \operatorname{diam}^{2}(Y)\right\}} \\
\max \{\operatorname{diam}(X), \operatorname{diam}(Y)\} & \leqslant \eta_{2} \operatorname{dist}(X, Y) \\
|R e(k)| \max \left\{\operatorname{diam}^{2}(X), \operatorname{diam}^{2}(Y)\right\} & \leqslant \max \left\{\eta_{2}, \eta_{3}(\operatorname{Im}(k)) \operatorname{dist}(X, Y)\right\} \operatorname{dist}(X, Y)
\end{aligned}
$$

The first condition, i.e., 10a corresponds to the choice of the sector in the directional approach, with $M_{X}$ and $M_{Y}$ respectively the centres of the clusters $X$ and $Y$, while $c$ is a unit vector defining the direction along which the wave is travelling. This is the purely directional condition, which is included in the choice of $C_{0}$ from condition (8) (see formula (4.5) from Lemma 4.1 page 15 in [12]).

Then, condition (10b) is similar to the standard admissibility condition (6). Furthermore, they are equivalent for clusters of similar size.

In this work we focus on the understanding of condition (10c) which is used to determine when a sparse approximation can be performed. (10c) is an adaptation of the $\mathcal{H}^{2}$ part of the admissibility condition for kernels with complex wavenumbers. Importantly, this condition reduces the set of admissible blocks compared to the standard admissible condition in $\mathcal{H}$-matrices. (10c) includes two criteria that we separate below, see (11) and [12. If $\operatorname{Im}(k)$ is large enough (we will explain what it means in the following), the condition (10c) becomes:

$$
|R e(k)| \max \left\{\operatorname{diam}^{2}(X), \operatorname{diam}^{2}(Y)\right\} \leqslant \eta_{3}(\operatorname{Im}(k)) \operatorname{dist}^{2}(X, Y)
$$

This criterion becomes less and less restrictive as the value of damping ratio $\alpha=\operatorname{Im}(k) / \operatorname{Re}(k)$ increases, meaning that it does not significantly reduce the set of admissible blocks compared to standard $\mathcal{H}$-matrices. On the other hand, if $\operatorname{Im}(k)$ is small (which includes the limiting case $\operatorname{Im}(k)=0$ ) then $10 \mathrm{c}$ simplifies to the standard condition for $\mathcal{H}^{2}$-matrices in the case without damping [11]

$$
|R e(k)| \max \left\{\operatorname{diam}^{2}(X), \operatorname{diam}^{2}(Y)\right\} \leqslant \eta_{2} \operatorname{dist}(X, Y)
$$

Finally, 10c reduces to $10 \mathrm{~b}$ if $|\operatorname{Re}(k)| \sim(\max \{\operatorname{diam}(X), \operatorname{diam}(Y)\})^{-1}$ and $\operatorname{Im}(k)$ is small.

Figure 5 illustrates on an example $\left(\eta_{2}=2, \eta_{3}=0.5, \operatorname{Re}\left(k_{s}\right)=18\right.$ and $\left.\operatorname{Im}\left(k_{s}\right)=0.3\right)$ how the two criteria (11) and (12) help realize condition (10c). We represent the different scenarios for various hypothetical distances $y=\operatorname{dist}(X, Y)$ and diameters $x=\max (\operatorname{diam}(X), \operatorname{diam}(Y))$ of the blocks. The blue curve shows the limit when criterion (12) becomes satisfied and the red curve the limit when criterion (11) becomes satisfied. Obviously, there are four scenarios:

- both criteria hold;

- only criterion (11) holds;

- only criterion (12) holds;

- no criterion holds;

Let's now see how (10c) works in practice, for different damping ratios $\alpha_{s}$. For all the remaining of this work, we define $\mathbb{G}$ as the $3 N_{c} \times 3 N_{c}$ matrix corresponding to the discretization of the $3 \mathrm{D}$ visco-elastodynamic Green's tensor at the $N_{c}$ discretization points. This matrix is further decomposed into nine submatrices $\left(\mathbb{G}_{\alpha \beta}\right)_{1 \leq \alpha, \beta \leq 3}$, each submatrix $\mathbb{G}_{\alpha \beta}$ corresponding to the discretization of the $G_{\alpha \beta}$-component of the Green's tensor. We consider a practical implementation on a sphere of radius $a=1$, resulting in a $10274 \times 10274 \mathbb{G}_{11}$ matrix after discretization. All the different blocks of the $\mathcal{H}$-matrix representation are represented by black squares. The blocks are thus admissible if the square is located above the blue curve or the red curve. As a first illustration, see Figure 6, we consider $N_{\text {leaf }}=100$ and four values of damping ratio: $\alpha_{s}=0.01,0.1,1$ and 100. The wavenumber is chosen to yield a fixed density of points of 10 points per wavelength, i.e., $k_{s} a=18$. We see that for a small damping ratio $\left(\alpha_{s}=0.01\right.$ and 0.1$)$, the condition (10c) is not satisfied at all. For a larger damping ratio $\alpha_{s}=1$, some blocks satisfy (11) but none of them satisfies (12). In that case, (10c) is equivalent to (11). Even if a greater number of blocks are admissible with respect to criterion (11) for $\alpha_{s}=100$, criterion 12 is never fulfilled. In 


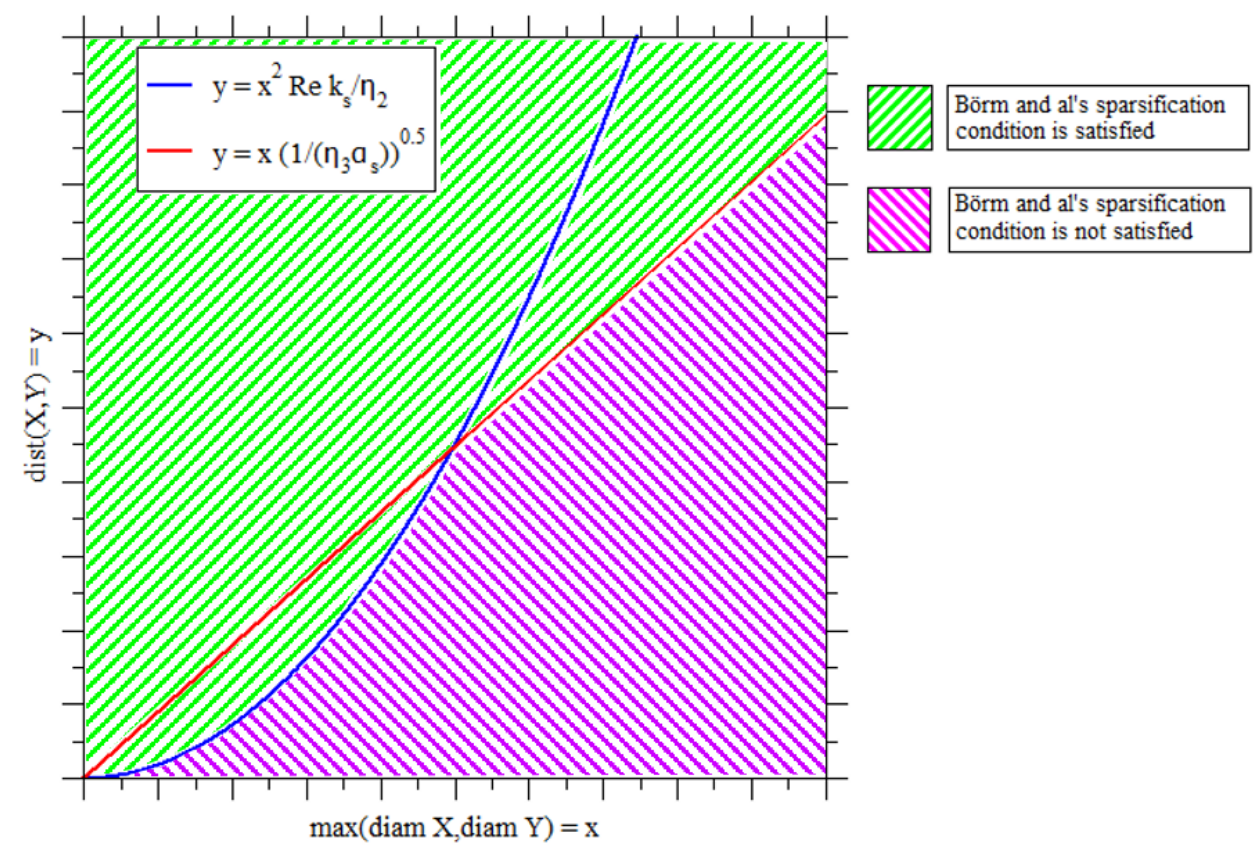

Fig. 5: Sparsification condition (10c): criterion (11) vs. criterion (12).

this setting, we conclude that condition (10c) is not satisfied when the damping ratio remains small. And, when the damping ratio increases, it seems that (11) is the dominant criterion to realize condition [10c). These results are in agreement with our expectation of a restrictive criterion for small damping ratios.

On the other hand, the fact that condition (10c) holds through (11) or (12) depends on the stopping criterion $N_{\text {leaf }}$ used in the binary tree. Indeed, we observe that if the value of $N_{\text {leaf }}$ is decreased, then one gets smaller clusters, i.e., with a smaller diameter. So, we consider the same same geometry, now with $N_{\text {leaf }}=20$. Figure 7 shows that for a small damping ratio $\alpha_{s}=0.01$, some blocks satisfy (12) but that none of them satisfies [11, so [10c) is equivalent to [12). For $\alpha_{s}=0.1$ some blocks satisfy both (12) and (11) criteria but (12) has become the dominant criterion. Then for $\alpha_{s}=1$, some blocks satisfy both (12) and (11) criteria, and (11) is the dominant criterion. Finally for $\alpha_{s}=100$, a majority of blocks satisfy (11) and there is a very small number of blocks which satisfy [12): (11) is the dominant criterion again. So, it appears that when $N_{\text {leaf }}$ is small, in the sense that a wider distribution of diameters towards 0 is at hand, the different scenarios expected in Figure 5 can actually be observed: condition $(10 \mathrm{c})$ is met even for a small damping ratio, and moreover either (11) or (12) is the dominant criterion.

It is also possible to get the same kind of results by decreasing the frequency in order to change the referenced position of both the blue curve and the red curve. Figure 8 presents the same results but when the frequency is divided by 10 , leading to about 100 points per wavelength. For the small damping levels $\alpha_{s}=0.01$ and $\alpha_{s}=0.1$, some blocks satisfy (12) but none of the blocks satisfy (11), so condition (10c) is equivalent to (12). For $\alpha=1$, some blocks satisfy (12) and (11), but the majority of blocks satisfy (12), thus (10c) is equivalent to (12). Finally for $\alpha_{s}=100$, some blocks satisfy (12) and (11), but the majority of blocks satisfy (11), thus $(10 \mathrm{c}$ ) is equivalent to 111 . As a conclusion, it appears from these tests that decreasing (respectively increasing) the frequency, and as a consequence the value of $\operatorname{Re}\left(k_{s}\right)$, leads to a wider (respectively tighter) parabola and to swap the dominant condition between (11) and (12).

At this point, it is important to sum up our understanding on $\mathcal{H}$-matrices for oscillatory kernels with a complex wavenumber. To our best knowledge, the only work on the subject in the literature is [12]. But this work is dedicated to the improvement of directional $\mathcal{H}^{2}$-matrices for oscillatory kernels with a complex wavenumber. The tests we have performed show that the proposed improvement is in fact to relax the third condition in this admissibility condition (the one that is specific to $\mathcal{H}^{2}$ matrices) according to the level of damping in the kernel. However, it appears that this adaptation can still be improved on two aspects since: 


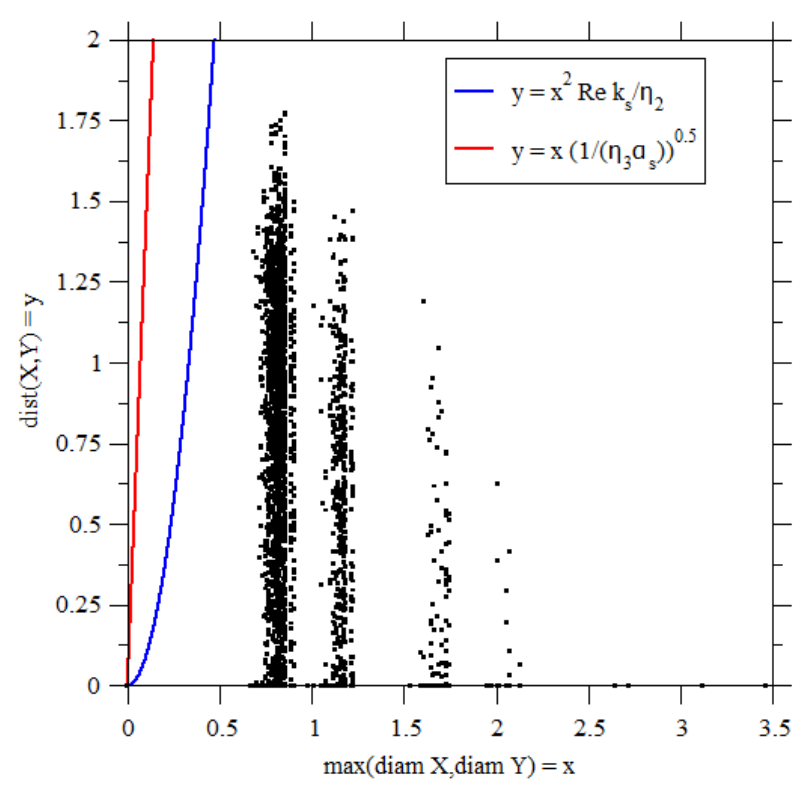

(a) $\alpha_{s}=0.01$

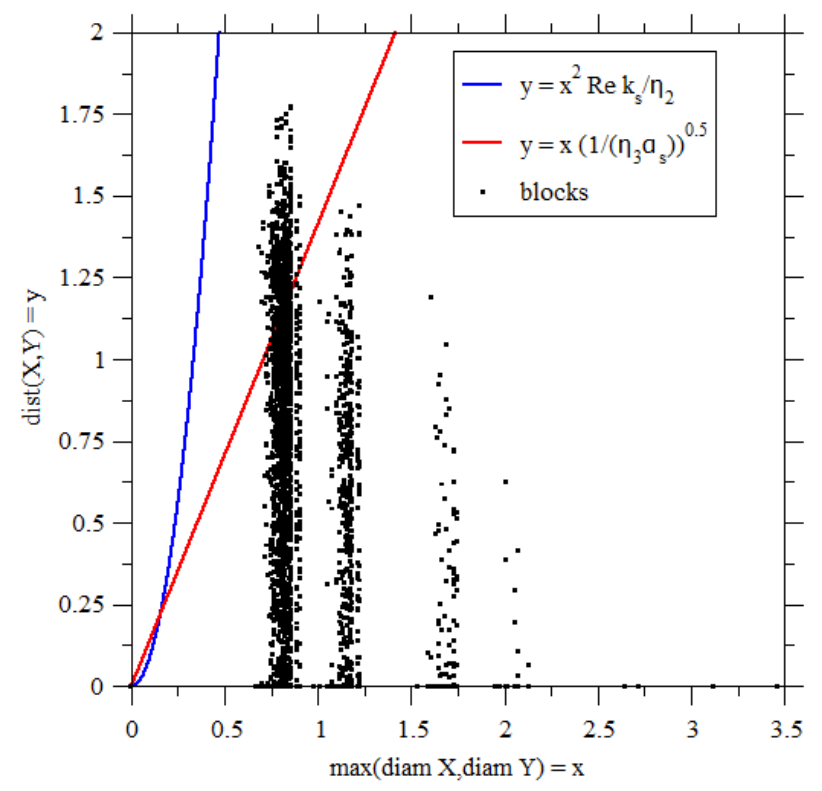

(c) $\alpha_{s}=1$

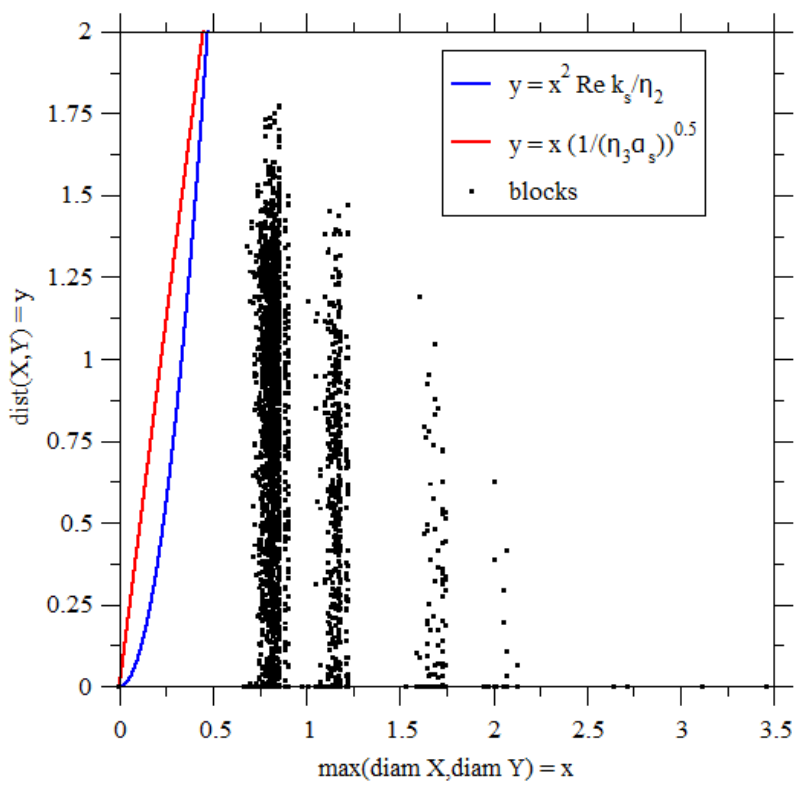

(b) $\alpha_{s}=0.1$

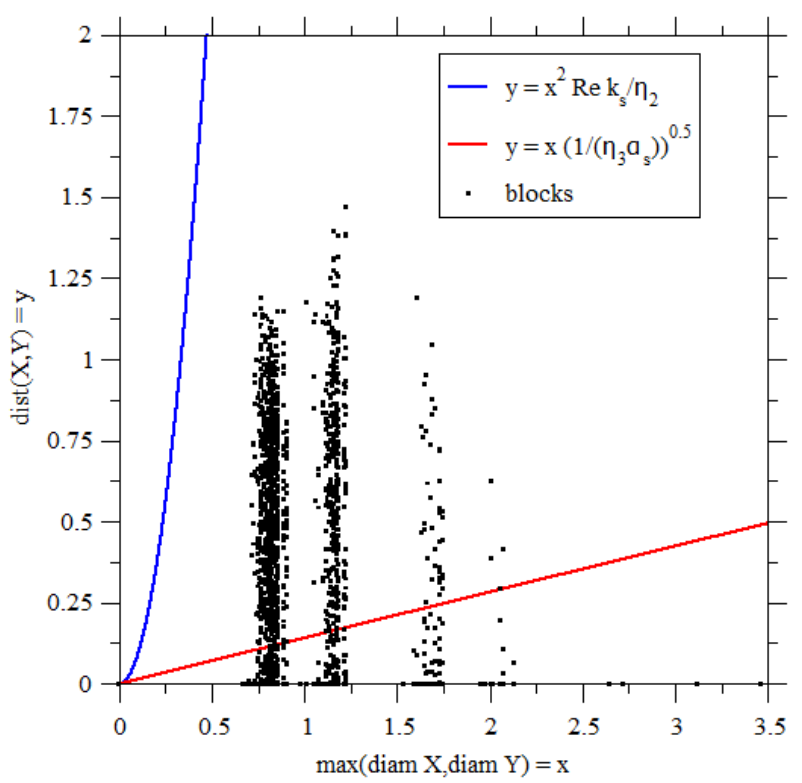

(d) $\alpha_{s}=100$

Fig. 6: Sparsification condition (10c): criterion [11) vs. criterion (12) for different damping ratios [ $\left.N_{\text {leaf }}=100 ; k_{s} a=18\right]$.

- its only aim is to relax the $\mathcal{H}^{2}$ part of the admissibility condition; In some sense it increases the number of admissible blocks; However it remains more restrictive than standard $\mathcal{H}$-matrices.

- it does not test non-admissible blocks to take advantage of the introduction of the complex wavenumber and thus one can not obtain further gain by compressing some of those blocks.

In the next Section, we perform extensive numerical tests to check if our new admissible condition enables to take these aspects into account and achieve better compression efficiency than $\mathcal{H}^{2}$-matrices. 


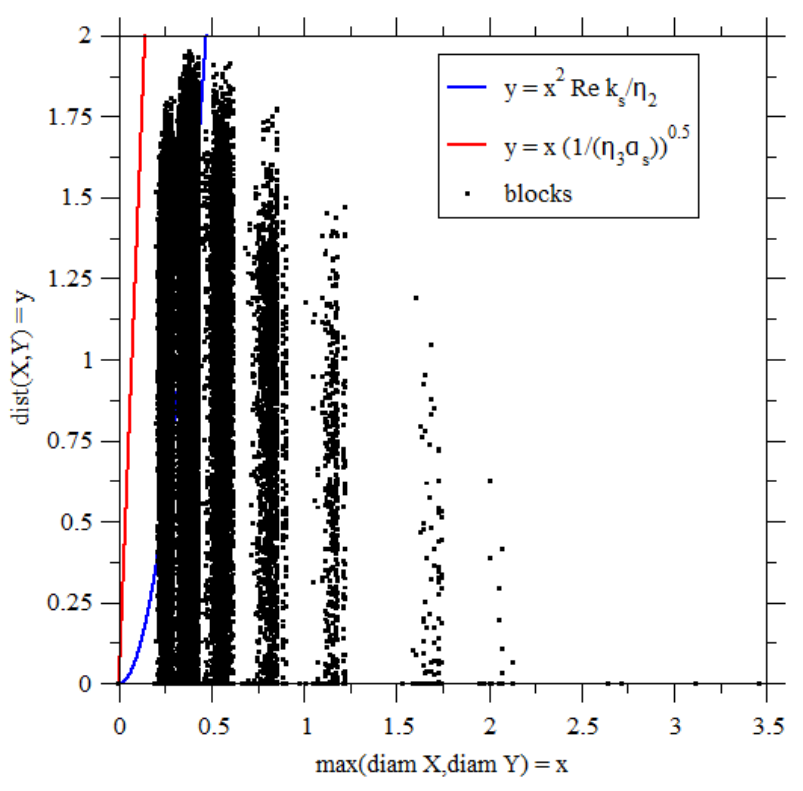

(a) $\alpha_{s}=0.01$

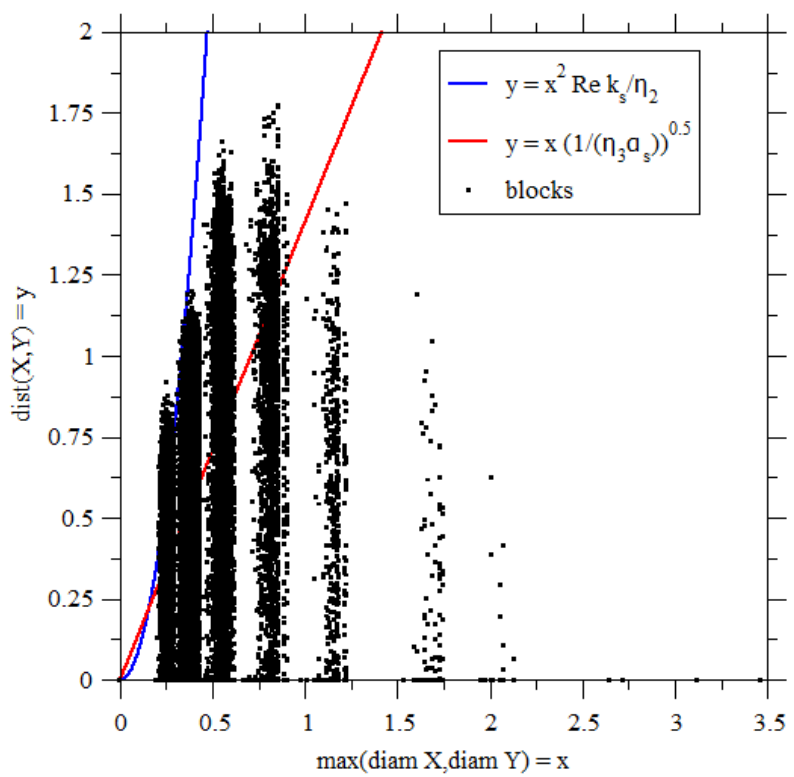

(c) $\alpha_{s}=1$

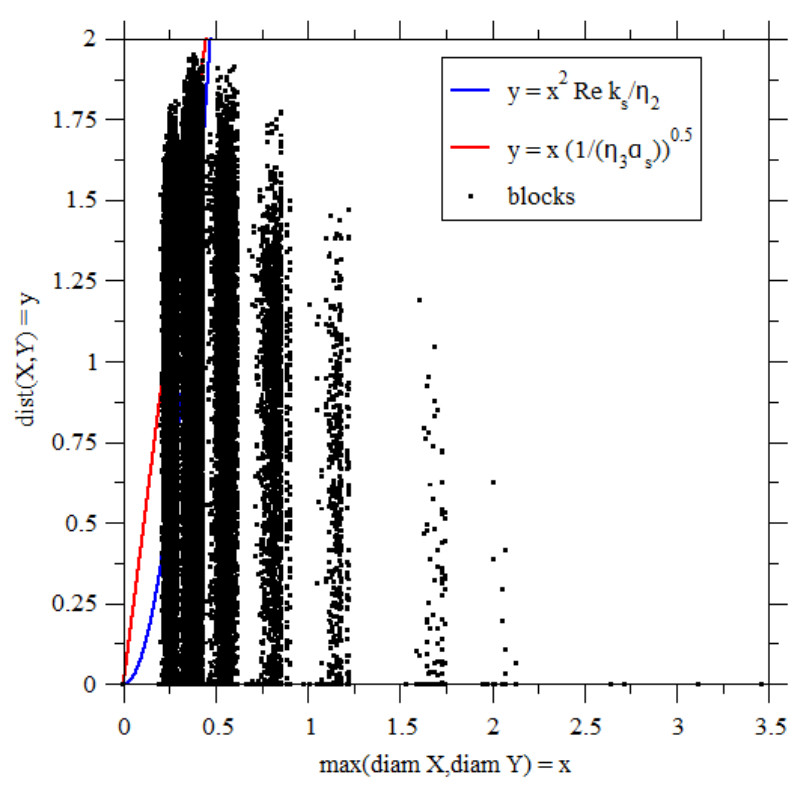

(b) $\alpha_{s}=0.1$

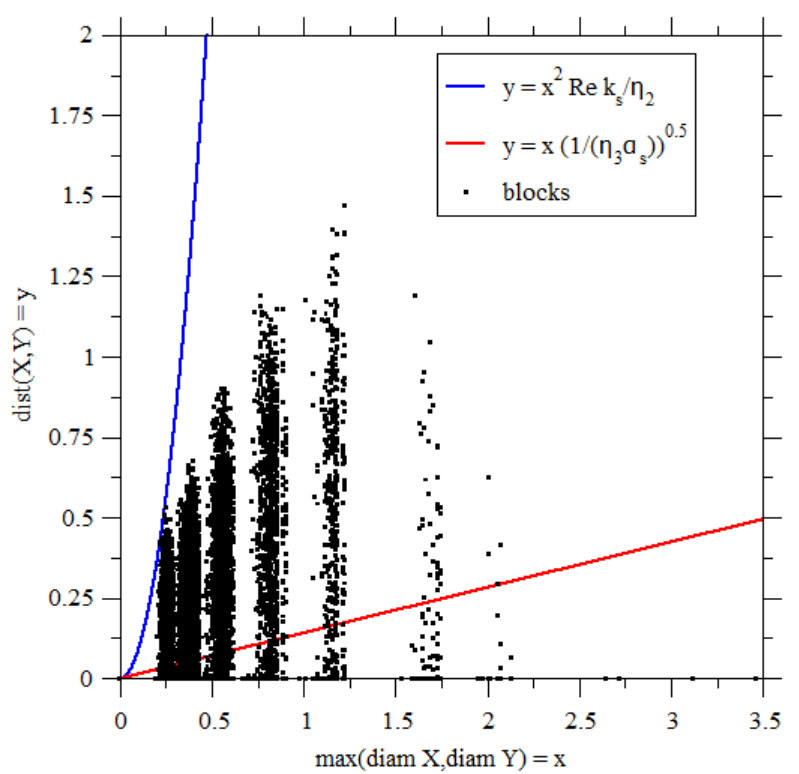

(d) $\alpha_{s}=100$

Fig. 7: Sparsification condition (10c): criterion (11) vs. criterion (12) for different damping ratios $\left[N_{\text {leaf }}=20 ; k_{s} a=18\right]$. 


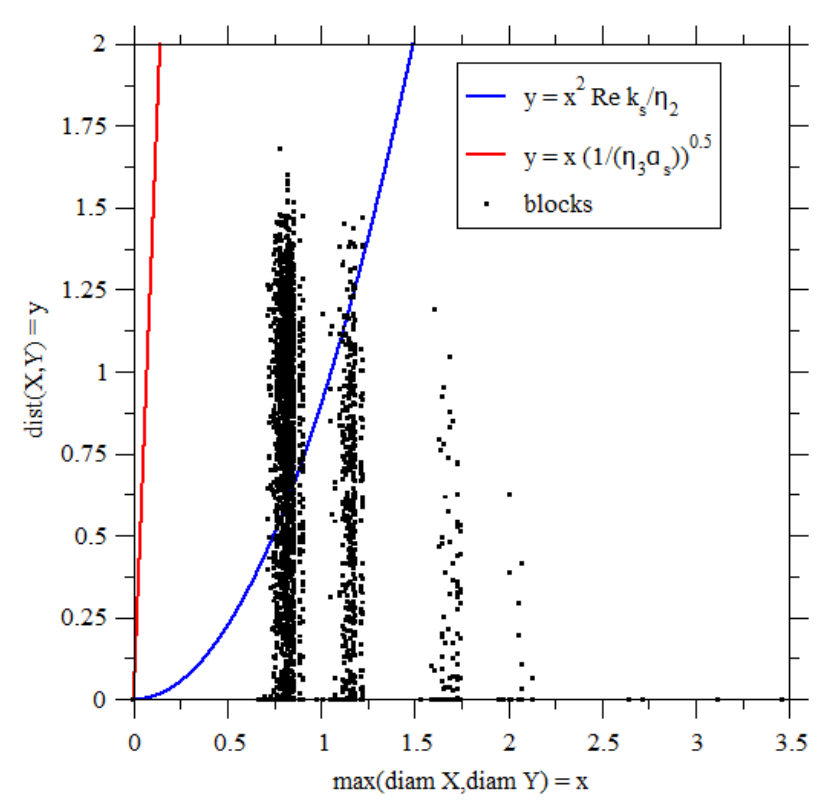

(a) $\alpha_{s}=0.01$

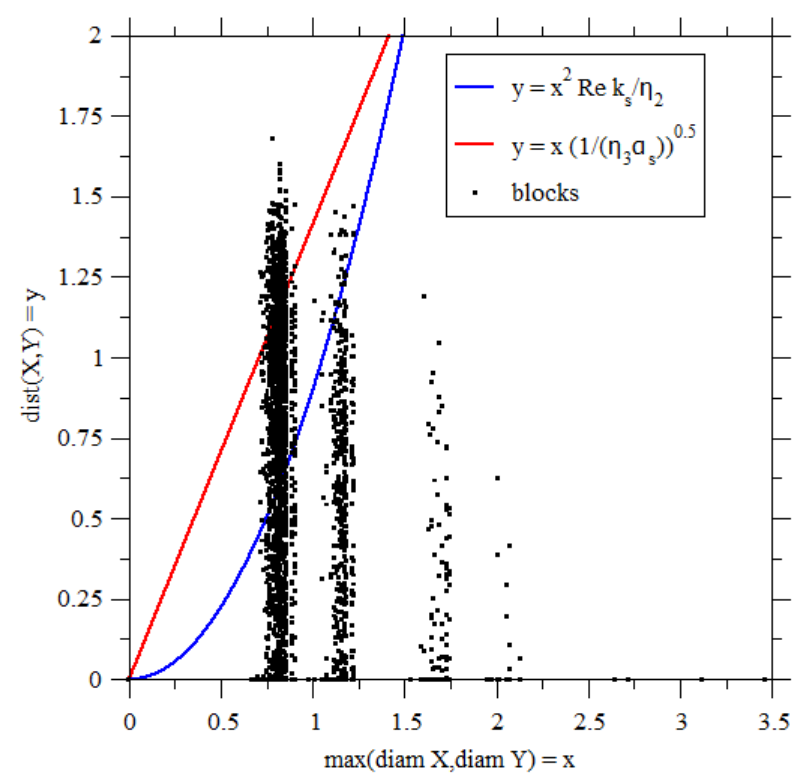

(c) $\alpha_{s}=1$

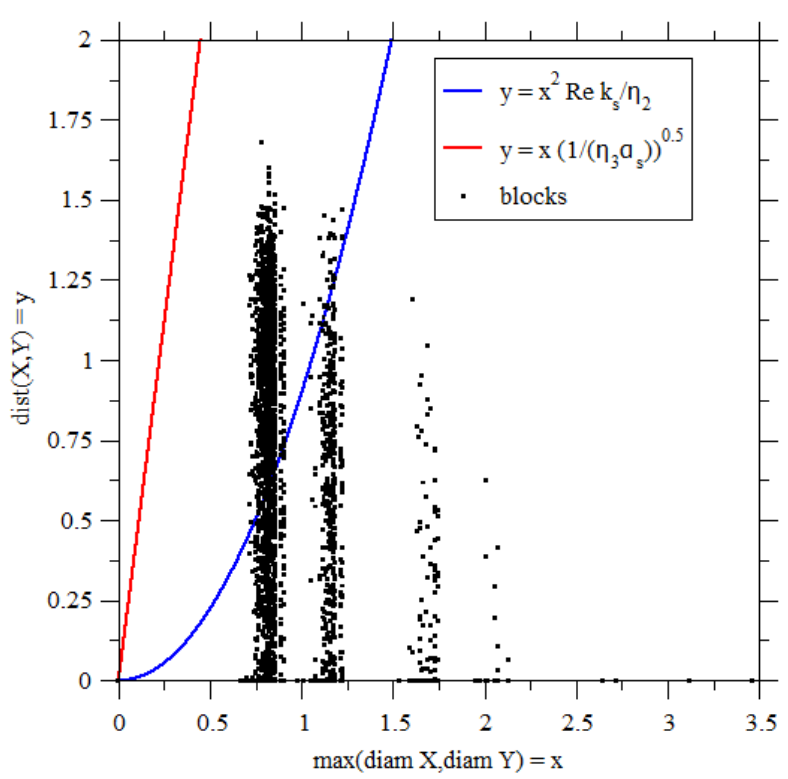

(b) $\alpha_{s}=0.1$

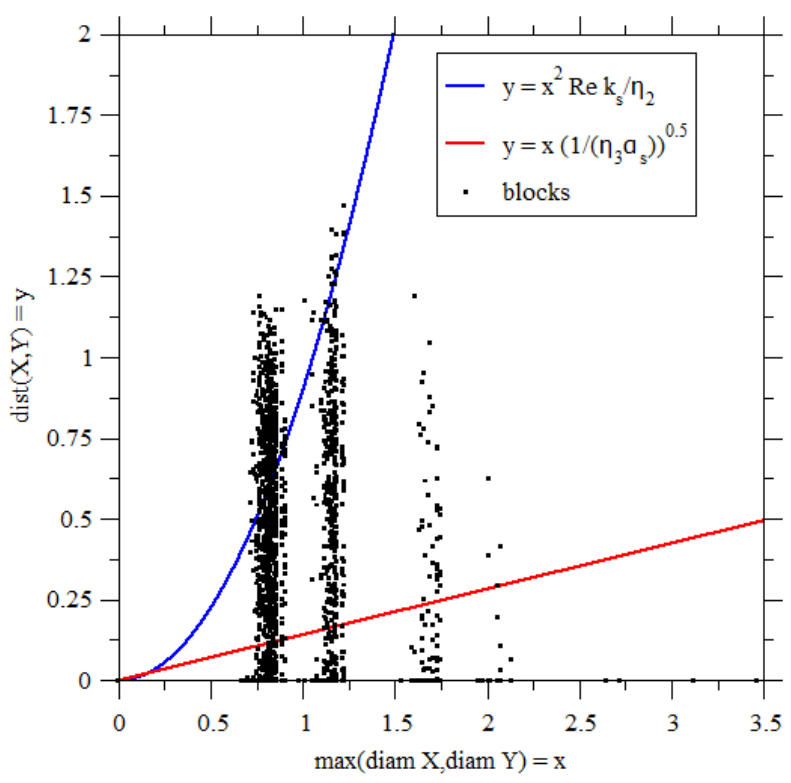

(d) $\alpha_{s}=100$

Fig. 8: Sparsification condition [10c): criterion (11) vs. criterion (12) for different damping ratios [ $\left.N_{\text {leaf }}=100, k_{s} a=1.80\right]$. 


\section{Efficiency of the new admissibility condition for standard $\mathcal{H}$-matrices}

In this section, numerical experiments are conducted to compare both admissibility conditions $(\sqrt[6)+(9)]{)}$ and their "counterpart" $(\sqrt{10 b}+(\sqrt{10 \mathrm{c}}))$. To that aim, we study a wave propagation problem in a viscoelastic media containing an obstacle represented by a sphere of radius $a=1$.

\subsection{Practical parameters for the sparsification condition from [12]}

The additional condition (8) where $\tilde{m}=-1$ enables to replace the admissible blocks by matrices of zeros in the approximated Green's tensor. By definition of $r_{\text {min }}$, we know that $r \geq r_{\min }$ in $(8)$. In the following we assume that $0<\varepsilon_{\text {error }} \leq r_{\text {min }}$. By applying the $\log$ on both sides of the inequality and "neglecting the constant", i.e., setting $C_{0} \rho_{0}$ to 1 , we obtain the sufficient condition:

$$
\log \left(\frac{1}{\varepsilon_{\text {error }}}\right) \frac{2}{\sigma r} \leq \operatorname{Im}(k)
$$

By sufficient we mean that:

- condition (8) and condition (13) are equivalent if $\varepsilon_{\text {error }} \sim r$;

- condition (13) implies condition (8) if $\varepsilon_{\text {error }} \ll r$.

We now choose the value of $\sigma$ to implement the condition 13 for our practical applications. To do that, we apply the condition (13) with $k=k_{s}$ and $\varepsilon_{\text {error }}=r_{m i n}$, in conjunction with the admissibility condition (10). The number of discretization points is $N_{c}=10274\left(\varepsilon_{\text {error }}=0.0253\right)$, and a frequency corresponding to $k_{s} a=18$ (in order to have roughly 10 points per wavelength). For a damping ratio $\alpha_{s}=100$, one finds by direct inspection that $r_{A C A}^{\max }=0$ (and then all admissible blocks can be replaced by matrices of zeros). Testing the condition (13) in the submatrix $\mathbb{G}_{11}$ for different values of $\sigma$ among $\left\{10^{-4}, 10^{-3}, \ldots, 0.5,1,2, \ldots, 10^{4}\right\}$, we find that it is satisfied for all admissible blocks and for all values of $\sigma$, except $10^{4}$. So, in the rest of the manuscript, we will keep the above condition with $\sigma=2$, which simplifies to

$$
\log \left(\frac{1}{\varepsilon_{\text {error }}}\right) \frac{1}{r} \leq \operatorname{Im}(k)
$$

as a practical implementation of the additional condition (8).

4.2 Practical choices and illustration of the new admissibility condition

The parameters used in all this section are given in Table 1 $\omega$ is chosen again to achieve about 10 points per wavelength $\lambda_{s}$.

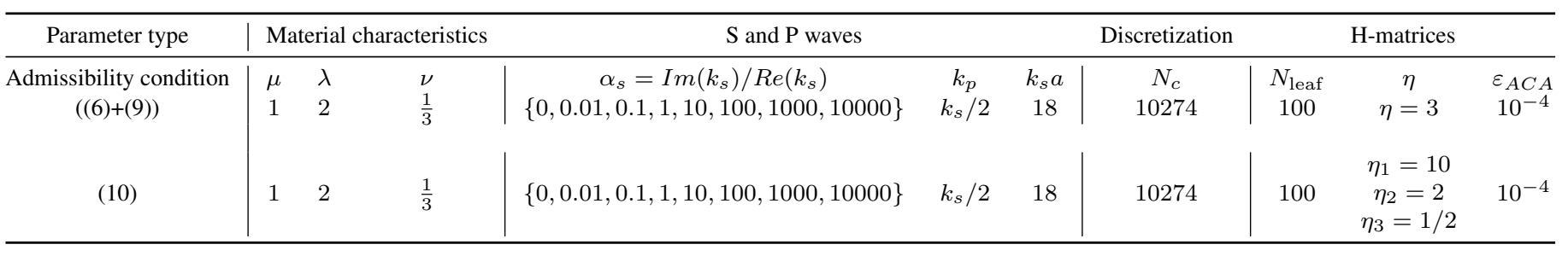

Table 1: Parameters.

The first parameter is $r_{\text {lim }}$ that appears in the condition (9). For a fixed value $\varepsilon_{\text {decay }}$ in condition (7), we are able to determine $r_{l i m}$ by observing the evolution of $\frac{\exp \left(-\operatorname{Im}\left(k_{p}\right) r\right) / r}{\exp \left(-\operatorname{Im}\left(k_{p}\right) r_{m i n}\right) / r_{\min }}$ with respect to $r$ for different values of $k_{p}$. Indeed, comparing condition (7) with $k=k_{p}$ and $k=k_{s}$, we observe that it is more restrictive with $k_{p}$ than with $k_{s}$, because $0<\operatorname{Im}\left(k_{p}\right)<\operatorname{Im}\left(k_{s}\right)$. We first conduct a numerical experiment to determine the influence on the overall accuracy of the tolerance $\varepsilon_{\text {decay }}$ in (7). We perform the analysis with the conditions $(\sqrt[6]{6}+(9))$ and

$$
\alpha_{s}=\operatorname{Im}\left(k_{s}\right) / \operatorname{Re}\left(k_{s}\right) \in\{10,11,12,13,14,15,17,20,33,66,100,1000,10000\}
$$

Let $\mathbb{G}_{a p p}$ be the approximated version of $\mathbb{G}$ in which the submatrices corresponding to admissible blocks are replaced by their low-rank approximations and/or by matrices of zeros in case condition 9) is also considered. Importantly, we are not 
interested in representing $\mathbb{G}$ accurately but by obtaining an accurate solution of a BEM problem. We introduce $V_{a l}$, a random vector whose values are drawn from the standard uniform distribution on $] 0,1[$. We will consider two relative errors:

$$
\begin{aligned}
e r r_{r e l_{1}} & =\frac{\left\|\mathbb{G}_{11}-\left(\mathbb{G}_{11}\right)_{a p p}\right\|_{\mathcal{L}^{2}}}{\left\|\mathbb{G}_{11}\right\|_{\mathcal{L}^{2}}} \\
e r r_{r e l_{2}} & =\frac{\left\|\left(\mathbb{G}_{11}-\left(\mathbb{G}_{11}\right)_{a p p}\right) V_{a l}\right\|_{\mathcal{L}^{2}}}{\left\|\mathbb{G}_{11} V_{a l}\right\|_{\mathcal{L}^{2}}}
\end{aligned}
$$

The first relative error might be too strict compared to the error observed in a BEM solver. It represents the upper bound of the error obtained when a matrix-vector product is performed in the iterative solver. The second relative error should be more representative for the "mean" error carried in the solution of the BEM elastodynamic problem. In Table 2, we report $\overline{\text { err }_{\text {rel }}}$ that is the average observed value over fifty values of $\mathrm{err}_{\mathrm{rel}}$ calculated with fifty random vectors $V_{a l}$.

In Table 2 we report these errors for three values of $\varepsilon_{\text {decay }}$. We note that as expected, it has an effect on the accuracy of the approximation. For $\varepsilon_{\text {decay }}=10^{-10}$, the relative error on the Green's tensor is always well below $10^{-3}$ for both $\mathrm{err}_{\mathrm{rel}}$ and $\overline{\mathrm{errel}_{2}}$. In comparison, the relative approximation errors reach a maximum of $\mathrm{err}_{\mathrm{rel}}$ and $\overline{\mathrm{err}_{\mathrm{rel}}}$ at about $1.510^{-2}$ for a tolerance $\varepsilon_{\text {decay }}=10^{-5}$ and about $310^{-2}$ for a tolerance $\varepsilon_{\text {decay }}=10^{-3}$. We performed the same tests on larger problems with $N_{c}$ roughly equal to respectively 30000,60000 and 100000 . In these cases, for $\varepsilon_{d e c a y}=10^{-10}$, both err $_{r e l_{1}}$ and $\overline{\mathrm{err}_{\mathrm{rel}}}$ are below $10^{-16}$. These results seem to advocate in favour of the use of $\varepsilon_{\text {decay }}=10^{-10}$. But the accuracy of the low-rank approximation is not the only factor to take into account. This criterion has also an influence on the number of blocks which can be replaced by matrices full of zeros and thus on the data compression rate. We will consider this aspect next.

\begin{tabular}{cccc}
\hline Damping ratio & $\begin{array}{c}\mathrm{err}_{\text {rel }_{1}} / \overline{\text { err rel }_{2}} \\
\varepsilon_{\text {decay }}=10^{-3}\end{array}$ & $\begin{array}{c}\text { err }_{\text {rel }} / \overline{e r r}_{\text {rel }} \\
\varepsilon_{\text {decay }}\end{array}=10^{-5}$ & $\begin{array}{c}\text { err }_{\text {rel }} / \overline{\text { err }}_{\text {rel }} \\
\varepsilon_{\text {decay }}\end{array}=10^{-10}$ \\
\hline 11 & $2.4810^{-2} / 2.5110^{-2}$ & $1.6610^{-4} / 1.6710^{-4}$ & $4.2510^{-10} / 4.2610^{-10}$ \\
12 & $2.8810^{-2} / 2.9310^{-2}$ & $2.0010^{-4} / 1.9810^{-4}$ & $1.4010^{-10} / 1.4010^{-10}$ \\
13 & $2.8110^{-2} / 2.8510^{-2}$ & $1.5110^{-4} / 1.4910^{-4}$ & $7.3210^{-9} / 6.7910^{-9}$ \\
14 & $2.7210^{-2} / 2.7710^{-2}$ & $1.1310^{-4} / 1.1110^{-5}$ & $2.7610^{-9} / 2.5610^{-9}$ \\
15 & $2.6310^{-2} / 2.6810^{-2}$ & $8.3910^{-5} / 8.2810^{-5}$ & $1.2210^{-9} / 1.1610^{-9}$ \\
17 & $2.4510^{-2} / 2.4910^{-2}$ & $1.1810^{-2} / 1.1910^{-2}$ & $1.1010^{-9} / 1.2310^{-9}$ \\
20 & $2.1610^{-2} / 2.2010^{-2}$ & $1.4110^{-2} / 1.4210^{-2}$ & $1.4810^{-9} / 1.4910^{-9}$ \\
33 & $1.0410^{-2} / 1.0510^{-2}$ & $9.1010^{-3} / 9.1110^{-3}$ & $1.6210^{-7} / 1.6610^{-7}$ \\
66 & $1.7210^{-3} / 1.6610^{-3}$ & $1.5510^{-3} / 1.5010^{-3}$ & $6.0910^{-4} / 6.1310^{-4}$ \\
100 & $3.5010^{-4} / 3.3810^{-4}$ & $3.4510^{-4} / 3.3310^{-4}$ & $3.4110^{-4} / 3.2910^{-4}$ \\
1000 & $<10^{-16}$ & $<10^{-16}$ & $<10^{-16}$ \\
10000 & $<16$ & $<10^{-16}$ & $<10^{-16}$ \\
\hline \multicolumn{5}{c}{}
\end{tabular}

Table 2: volution of $\mathrm{err}_{\mathrm{rel}}$ and $\mathrm{err}_{\mathrm{rel}}$ with respect to the damping ratio and tolerance in condition (7) [ $\left.k_{s} a=18\right]$.

Now that we have an insight on the effect of $\varepsilon_{\text {decay }}$ on the accuracy, Figure 9 illustrates the behavior of the dominant factor in the viscoelastodynamic Green's tensor. This Figure shows in particular that, as expected, if the damping ratio increases the required value of $r_{l i m}$ decreases.

We now compare the effect of the additional condition (9) to complement the classic admissibility condition (6) on the submatrix $\mathbb{G}_{11}$. On Figure 10 , admissible (and zero) blocks are in blue and red blocks represent non-admissible blocks. In comparison, Figure 10p show the distribution if only condition (6) is enforced. A significant gain of storage is clearly visible as the number of red blocks is quite smaller in Figure $10 \mathrm{p}$ than in Figure $10 \mathrm{p}$. The observed data compression rates are $\tau=0.104$ with the conditions $(6+6)$ to be compared to $\tau=0.202$ with the standard condition (6).

We illustrate in Figure 11 three different tolerances $\varepsilon_{\text {decay }}$ for $\alpha_{s}=15$ to show that the difference in the storage is not very significant. In Table 3 we gather the data compression rates for different tolerances $\varepsilon_{\text {decay }}$ and damping ratios $\alpha_{s}$. Note that in all the following tables (Table 3 to Table 8), compression rates are given in thousandths for more readability. In practice, the tolerance has a very moderate influence on the data compression rate in this exhaustive comparison. Moreover, the best result is almost always achieved with its smallest value. Based on these observations, we set $\varepsilon_{\text {decay }}=10^{-10}$ in the following. 


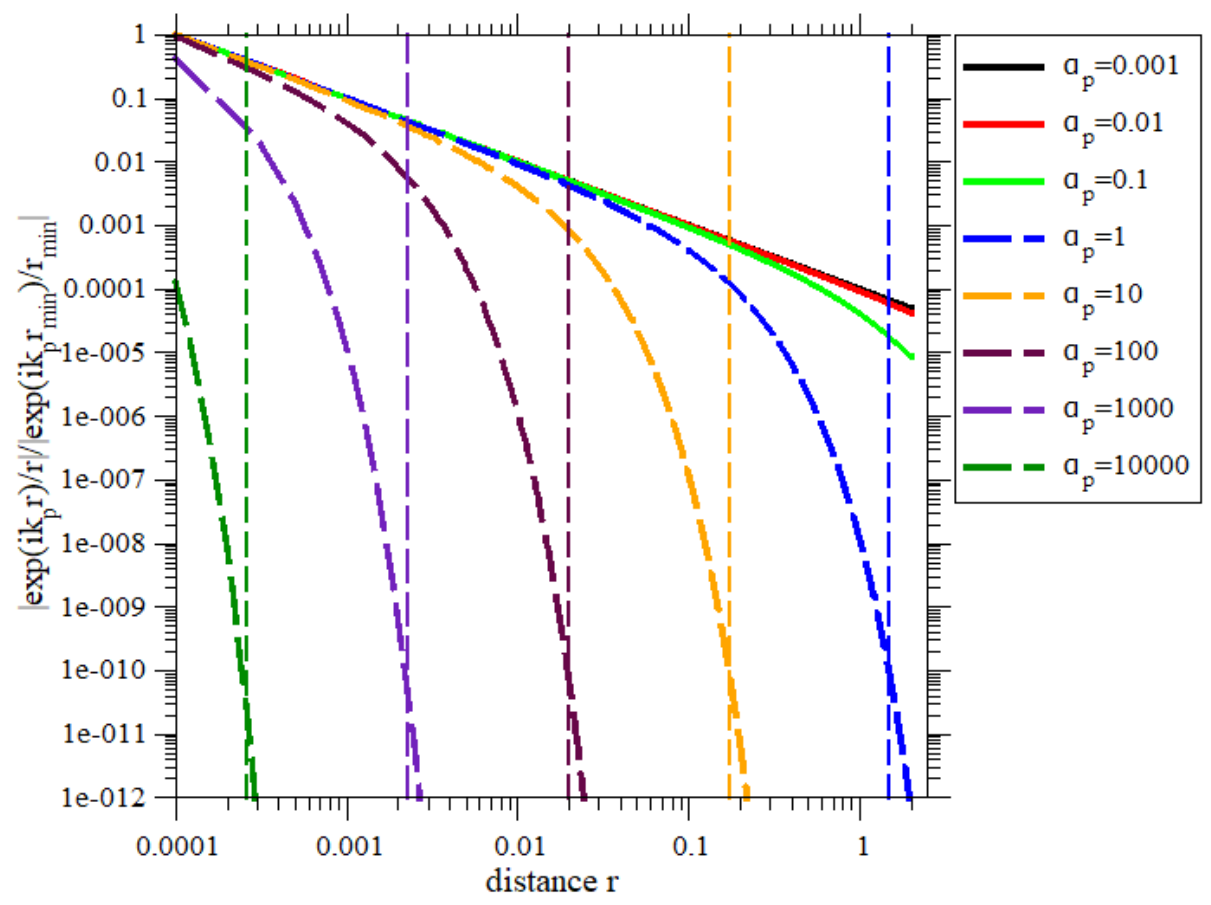

Fig. 9: Evolution of the dominant factor in the visco-elastodynamic Green's tensor with respect to the distance for tolerance $\varepsilon_{\text {decay }}=10^{-10}$ and different damping ratios $\left[k_{p} a=k_{s} a / 2=9\right]$.

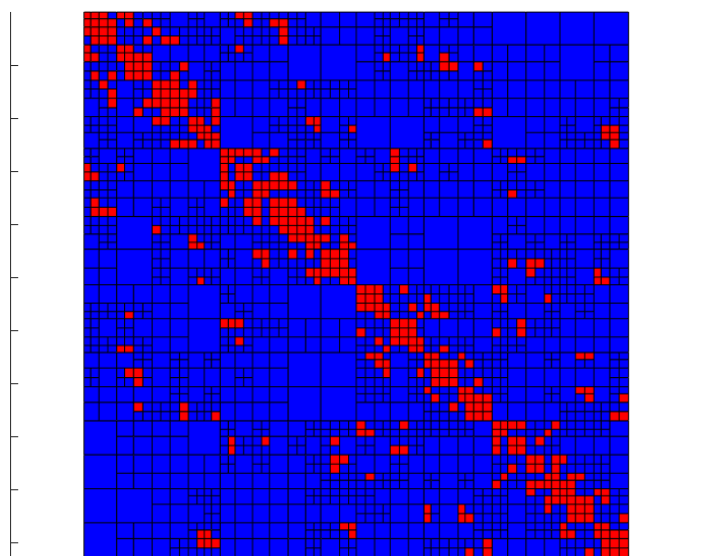

(a) Improved condition $-\tau=0.104$

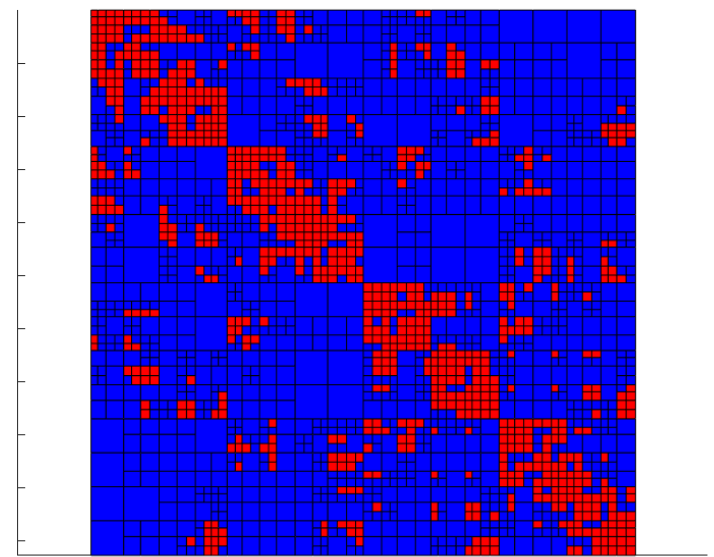

(b) Standard condition - $\tau=0.202$

Fig. 10: Illustration with coloured patches of the type of blocks represented when (a) conditions $(\sqrt{6})+(9)$ ) are enforced and (b) when only (6) is applied with a damping ratio $\alpha_{s}=100$; tolerance is set to $\varepsilon_{\text {decay }}=10^{-10}\left[k_{s} a=18\right]$. 


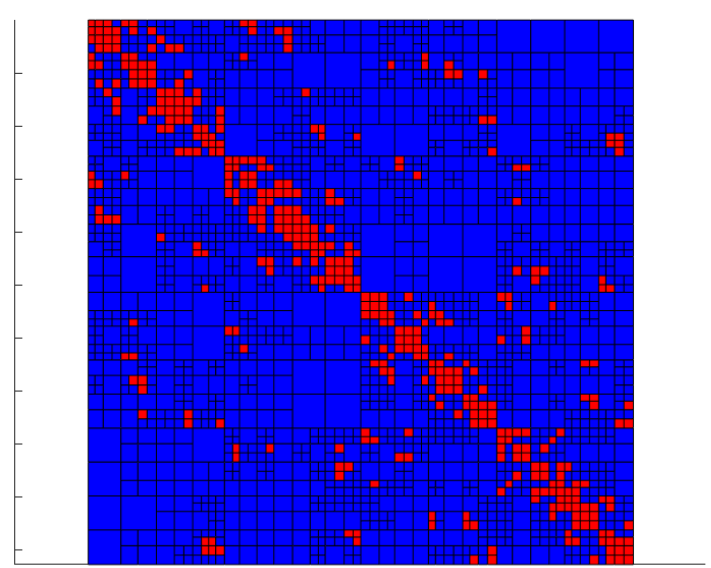

(a) $\varepsilon_{\text {decay }}=10^{-3}-\tau=0.103$

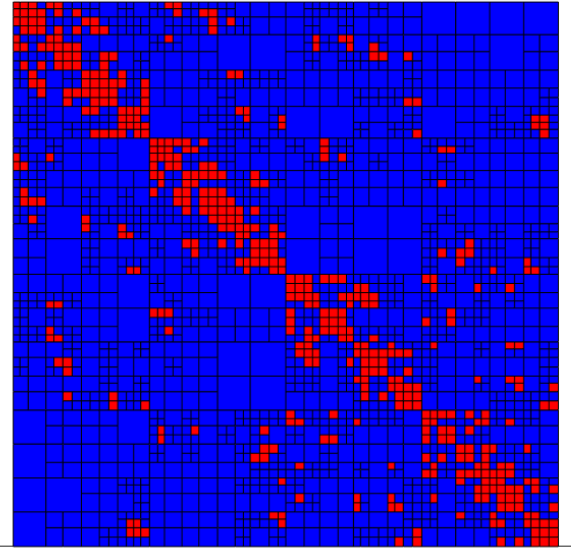

(b) $\varepsilon_{\text {decay }} 10^{-5}-\tau=0.118$

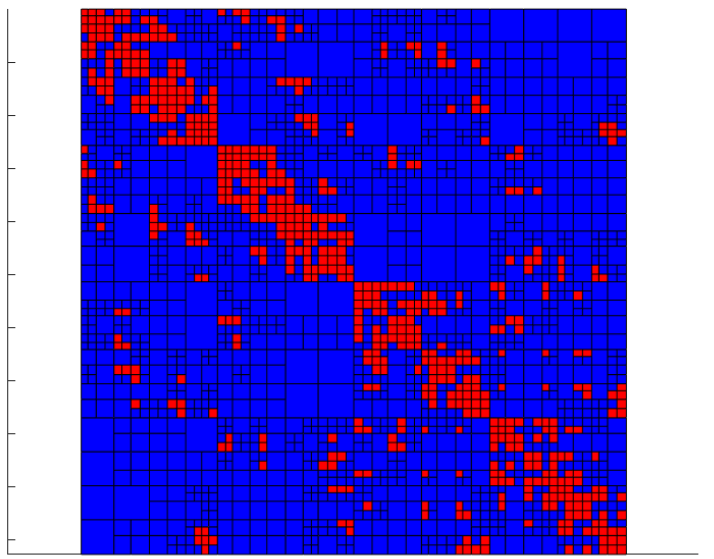

(c) $\varepsilon_{\text {decay }}=10^{-10}-\tau=0.147$

Fig. 11: Illustration with coloured patches of the type of blocks represented when conditions $((6)+(9))$ are applied for a damping ratio $\alpha_{s}=15$ and different tolerances $\varepsilon_{\text {decay }}\left[k_{s} a=18\right]$.

\begin{tabular}{|c|c|c|c|c|}
\hline Damping ratio $\alpha_{s}$ & $\begin{array}{l}\tau \text { with } \\
6 \text { (6) only }\end{array}$ & $\begin{array}{c}\tau \text { with }[6+9] \\
\varepsilon_{\text {decay }}=10^{-3}\end{array}$ & $\begin{array}{l}\tau \text { with } 6+9] \\
\varepsilon_{\text {decay }}=10^{-5}\end{array}$ & $\begin{array}{c}\tau \text { with } 6+9] \\
\varepsilon_{\text {decay }}=10^{-10}\end{array}$ \\
\hline 0 & 317 & 317 & 317 & 317 \\
\hline 0.01 & 317 & 317 & 317 & 317 \\
\hline 0.1 & 315 & 315 & 315 & 315 \\
\hline 1 & 292 & 292 & 292 & 292 \\
\hline 10 & 202 & 202 & 202 & 202 \\
\hline 15 & 202 & 103 & 118 & 147 \\
\hline 100 & 202 & 99.9 & 101 & 104 \\
\hline 1000 & 202 & 95.4 & 96.5 & 97.5 \\
\hline 10000 & 202 & 94.9 & 94.9 & 94.9 \\
\hline
\end{tabular}

Table 3: Evolution of the data compression rate $\tau$ (expressed in thousandths for more readability) with respect to the damping ratio and to the tolerance in condition (7) $\left[k_{s} a=18\right]$. 


\subsection{Influence of the condition (10c)}

Now that all the practical choices have been made for the parameters, numerical experiments are conducted to compare both admissibility conditions $(\sqrt{6}+\sqrt{9})$ ) and their more involved "counterpart" $((10 \mathrm{~b})+(\sqrt{10 \mathrm{c}}))$. The present objective is to investigate whether the additional admissibility condition (9) leads to better, or worse, data compression, compared to the conditions $(10 \mathrm{~b})+10 \mathrm{c})$ and $(14)_{k=k_{s}}$.

The parameters used in this section are gathered in the Table 1 . We use $\eta_{2}=2$ as it is the optimal choice proposed in [10] and $\eta=3$ as it is the optimal choice of $\eta$ resulting from the analysis detailed in [14].

First we consider the conditions $(\sqrt{6}+(9)$ ). Figure 12 illustrates the evolution of the data sparse approximation (and gives the corresponding data compression rate $\tau$ ) for different values of the damping ratio $\alpha_{s}$. We observe as expected that the compression improves with the increase of the damping ratio. This is explained by the decrease of the value of $r_{l i m}$, cf. condition (7) (see Fig 9).

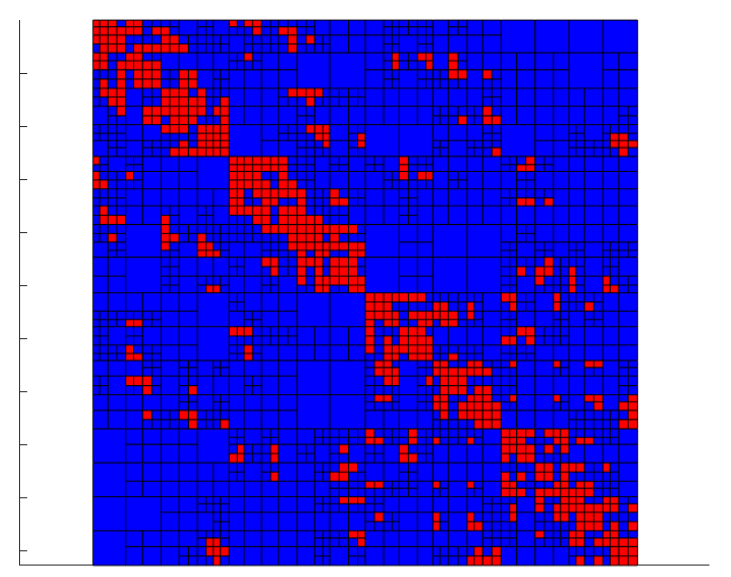

(a) $\alpha_{s}=15-\tau=0.147$

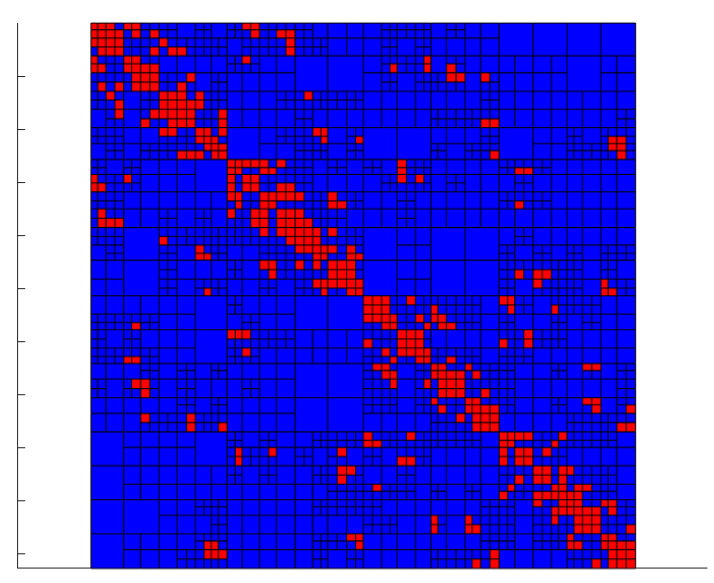

(b) $\alpha_{s}=100-\tau=0.104$

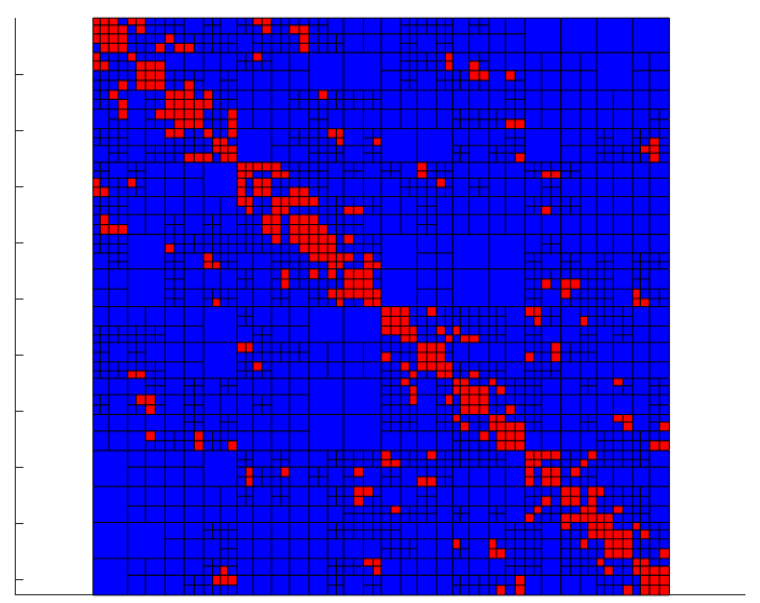

(c) $\alpha_{s}=1000-\tau=0.098$

Fig. 12: Illustration with coloured patches of the type of blocks represented when conditions $(6+(9)$ are applied for $\varepsilon_{\text {decay }}=10^{-10}$, with different damping ratios $\alpha_{s}\left[k_{s} a=18\right]$.

Now we consider the same configuration but use the more involved criterion $(\sqrt{10 b})+(10 \mathrm{c})+(14))$. Figure 13 shows the results for $\eta_{2}=2$, while Figure 14 shows the results for $\eta_{2}=3$. This latter case (see (10b) is similar to the application of the classical admissibility condition (6). It allows us to see more precisely the influence of $(10 \mathrm{c})+(14)$. By comparing Figures $10 \mathrm{p}$ and $14 \mathrm{p}$, it seems that $\eta_{2}=3$ enables to have similar results between $(6)$ and $((10 \mathrm{~b})+(10 \mathrm{c})+(14))$. To confirm 
these results, Table 4 gives the data compression rates for various admissibility conditions and damping ratio $\alpha_{s}$. It appears that the condition $((10 \mathrm{~b})+(10 \mathrm{c})+(14))$ does not give results better than the ones obtained with (6).

To conclude, and based on all the previous numerical studies, it appears that the use of (9) in addition to the classical condition (6) enables important storage reductions compared to the other approaches. These encouraging results on the efficiency of standard $\mathcal{H}$-matrices can be easily explained. Comparing the involved $\mathcal{H}^{2}([10 \mathrm{~b})+(10 \mathrm{c})+(14))$ condition with the improved, yet simple $\mathcal{H}(\sqrt{6}+\sqrt{9})$ condition, we emphasize two important aspects:

- The set of admissible blocks in the $\mathcal{H}^{2}$ case is, by definition, a subset of the set of admissible blocks in the $\mathcal{H}$ case. This restriction is based on an a priori study, specifically designed to exclude blocks which are not low-rank. However, it appears in the case with a complex wavenumber that the standard $\mathcal{H}$ condition is already efficient and does not need to be restricted. Otherwise it leads to a less efficient approach.

- The proposed improved $\mathcal{H}$ condition further reduces the storage requirements by approximating by blocks full of zero not only the admissible blocks but also a portion of non-admissible blocks (in the traditional sense of admissibility).

Due to its high efficiency and ease of implementation we advocate the use of standard "improved" $\mathcal{H}$-matrices for problems with an oscillatory kernel and a complex wavenumber. In the next Section, we illustrate the practical efficiency of the proposed approach for two possible configurations: visco-elastodynamic problems in the frequency-domain and purely elastic problems in the time-domain.

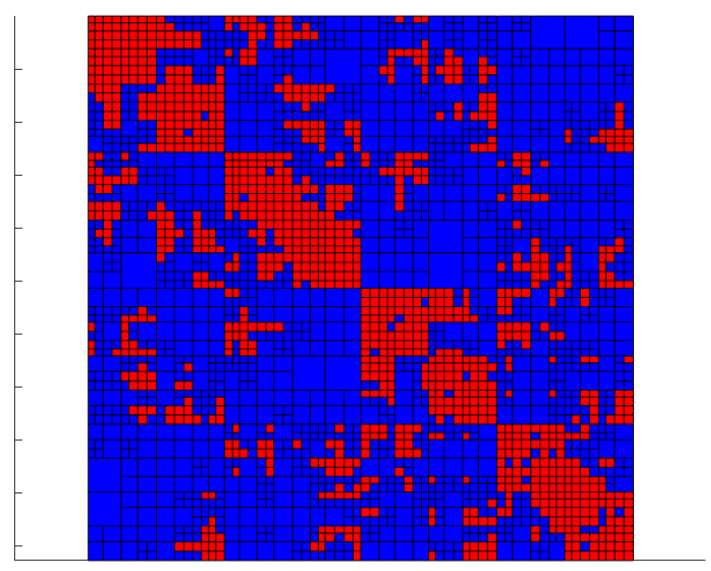

(a) $\alpha_{s}=15-\tau=0.278$

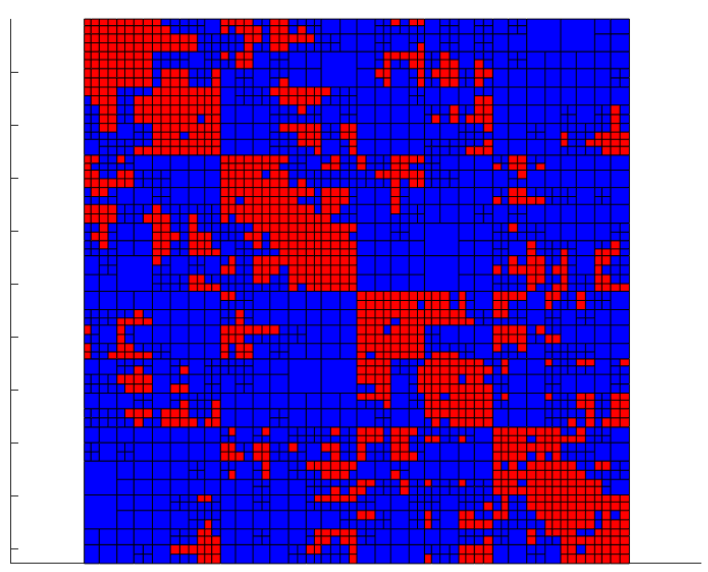

(b) $\alpha_{s}=100-\tau=0.278$

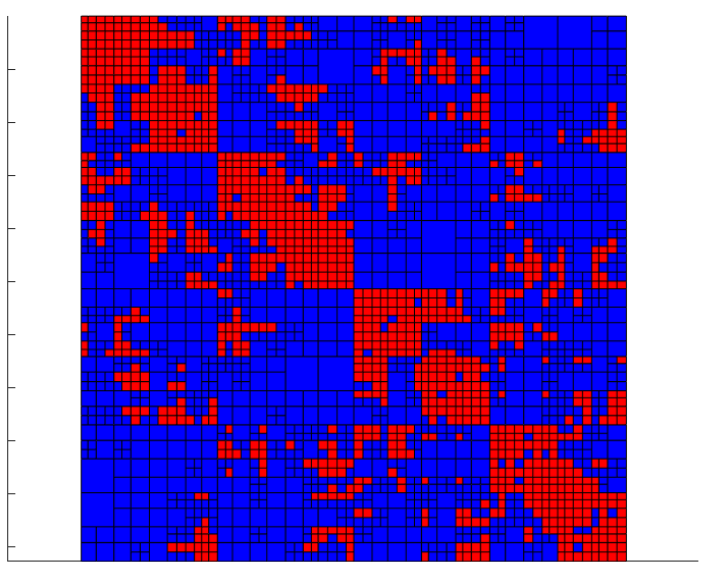

(c) $\alpha_{s}=1000-\tau=0.278$

Fig. 13: Illustration with coloured patches of the type of blocks represented when conditions (10b) and (10c) are applied with $\eta_{2}=2$ for different damping ratios $\alpha_{s}\left[k_{s} a=18\right]$. 


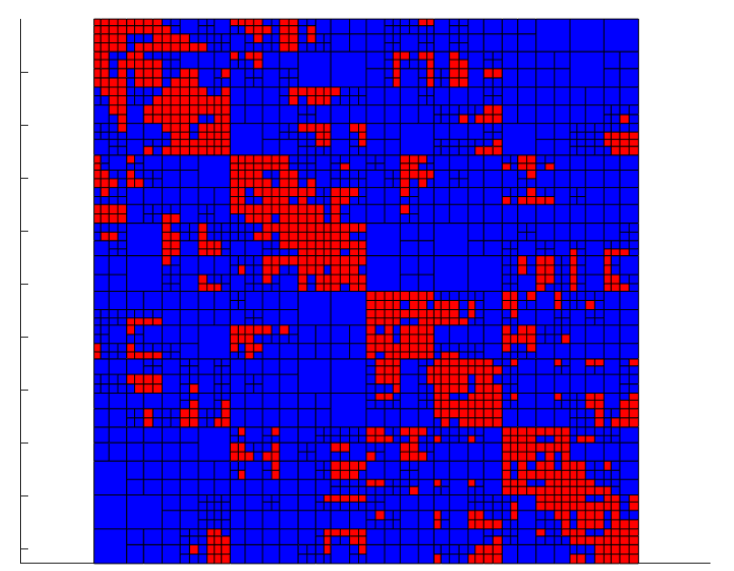

(a) $\alpha_{s}=15-\tau=0.222$

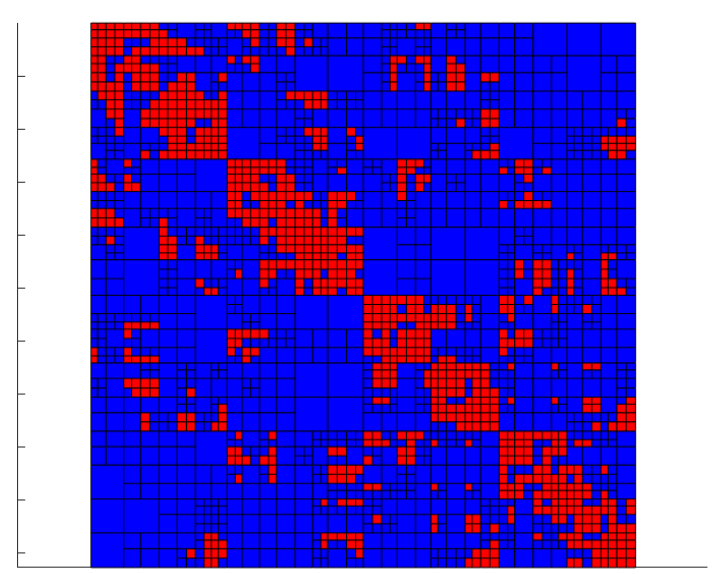

(b) $\alpha_{s}=100-\tau=0.209$

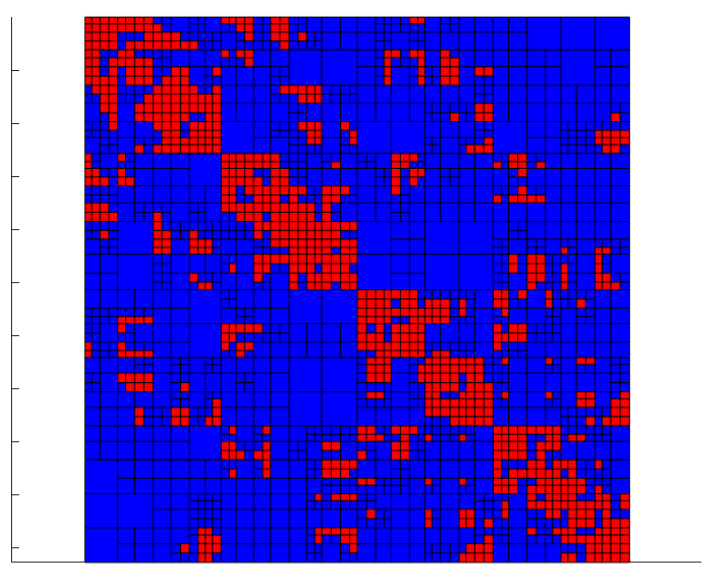

(c) $\alpha_{s}=1000-\tau=0.209$

Fig. 14: Illustration with coloured patches of the type of blocks represented when conditions (10b) and (10c) are applied with $\eta_{2}=3$ for different damping ratios $\left[k_{s} a=18\right]$.

\begin{tabular}{|c|c|c|c|c|}
\hline Damping ratio $\alpha_{s}$ & $\begin{array}{l}\tau \text { with } \\
\text { 6) only }\end{array}$ & $\begin{array}{c}\tau \text { with } 6]+9] \\
\varepsilon_{\text {decay }}=10^{-1}\end{array}$ & $\tau$ with $\frac{10 \mathrm{~b}}{\eta_{2}=2}+10 \mathrm{c}$ & $\tau$ with $\frac{10 \mathrm{~b}}{\eta_{2}=3}+10 \mathrm{c}$ \\
\hline 0 & 317 & 317 & 1000 & 1000 \\
\hline 0.01 & 317 & 317 & 1000 & 1000 \\
\hline 0.1 & 315 & 315 & 1000 & 1000 \\
\hline 1 & 292 & 292 & 799 & 799 \\
\hline 10 & 202 & 202 & 278 & 254 \\
\hline 15 & 202 & 147 & 278 & 222 \\
\hline 100 & 202 & 104 & 278 & 209 \\
\hline 1000 & 202 & 97.5 & 278 & 209 \\
\hline 10000 & 202 & 94.9 & 278 & 209 \\
\hline
\end{tabular}

Table 4: Evolution of the data compression rate $\tau$ (expressed in thousandths for more readability) with respect to the damping ratio and parameter $\eta_{2}\left[k_{s} a=18\right]$. 


\section{Application in two configurations: visco-elastodynamic BEMs and Z-BEMs}

\subsection{Efficiency in the context of visco-elastodynamic BEMs}

We consider first the case with a physical attenuation. We can write the constitutive equation for visco-elasticity as the convolution product of the relaxation tensor and the strain rate:

$$
\boldsymbol{\sigma}(\mathbf{x}, t)=\mathbf{C}(t) * \dot{\varepsilon}(\mathbf{x}, t)=\dot{\mathbf{C}}(t) * \varepsilon(\mathbf{x}, t)
$$

For a homogeneous isotropic visco-elastic medium, the relaxation tensor $C_{i j k l}(t)$ is written in terms of two independent Lamé-type coefficients:

$$
C_{i j k l}(t)=\left[\lambda(t) \delta_{i j} \delta_{k l}+\mu(t)\left(\delta_{i k} \delta_{j l}+\delta_{i l} \delta_{j k}\right)\right] H(t)
$$

where $H($.$) is a Heaviside step function. The rewriting of the visco-elastic constitutive law in the frequency domain, see [17]$ for more details, leads to:

$$
\sigma_{i j}(\mathbf{x}, \omega)=\hat{C}_{i j k l}(\omega) \varepsilon_{k l}(\mathbf{x}, \omega), \quad \hat{C}_{i j k l}(\omega)=\hat{\lambda}(\omega) \delta_{i j} \delta_{k l}+\hat{\mu}(\omega)\left(\delta_{i k} \delta_{j l}+\delta_{i l} \delta_{j k}\right)
$$

where $\hat{\lambda}(\omega)$ and $\hat{\mu}(\omega)$ are respectively the Fourier transforms of $\lambda(t) H(t)$ and $\mu(t) H(t)$. This highlights the analogy between the linear visco-elastic and the linear elastic time-harmonic configurations. Equality (19) shows that the main difference between the two situations is that the Lamé coefficients and consequently wave velocities and wavenumbers are complexvalued and frequency-dependent in the visco-elastic case. Following [18], we consider complex wavenumbers of the form:

$$
\hat{k}^{2}(\omega)=\frac{\rho \omega^{2}}{\hat{M}}
$$

where $\hat{M}(\omega)=M_{r}(\omega)-i M_{i}(\omega)$, with $M_{r}>0$ and $M_{i} \geq 0$, corresponds either to $\hat{M}=\hat{\lambda}$ or $\hat{\mu}$. These complex wavenumbers can be written as:

$$
\begin{aligned}
\hat{k}(\omega) & =\omega \sqrt{\frac{\rho\left(|\hat{M}|+M_{r}\right)}{2|\hat{M}|^{2}}}+i \omega \sqrt{\frac{\rho\left(|\hat{M}|-M_{r}\right)}{2|\hat{M}|^{2}}} \\
& =\omega \sqrt{\frac{\rho\left(|\hat{M}|+M_{r}\right)}{2|\hat{M}|^{2}}}\left(1+i \sqrt{\frac{|\hat{M}|-M_{r}}{|\hat{M}|+M_{r}}}\right)=\operatorname{Re}(\hat{k}(\omega))(1+i \alpha(\omega))
\end{aligned}
$$

$\alpha(\omega)$ corresponds to the ratio of the imaginary part over the real part of the complex wavenumber for physical configuration in case of real soils. We denote $Q^{-1}$ the damping coefficient associated with the physical material attenuation given by

$$
Q^{-1}=\frac{M_{i}(\omega)}{M_{r}(\omega)}
$$

It can be defined through empirical models, e.g., rheological models (Maxwell, Kelvin-Voigt, Zener) which are able to give the frequency-depend expression of the equivalent visco-elastic modulus (see [35] for more details on rheological models). Rewriting the expression of the attenuation factor $\alpha(\omega)$ as a function of $Q^{-1}$ we obtain:

$$
\alpha(\omega)=\sqrt{\frac{\left(\sqrt{1+\left(Q^{-1}\right)^{2}}-1\right)}{\left(\sqrt{1+\left(Q^{-1}\right)^{2}}+1\right)}}
$$

Damping in real soils follows the weak-dissipation assumption such as we could consider $Q^{-1} \ll 1$. Thus, truncating the Taylor of $\alpha$ at the first order yields to the approximation under the weak-dissipation configuration:

$$
\alpha \simeq \frac{1}{2} Q^{-1}
$$

Since $\hat{M}$ in 20, can be equal to $\hat{\lambda}$ or $\hat{\mu}$, and given 21] it follows that under the weak-dissipation assumption

$$
\hat{\mu}=\operatorname{Re}(\hat{\mu})\left(1-2 i \alpha_{\mu}\right) \text { and } \hat{k}_{s}=\omega \sqrt{\frac{\rho}{\operatorname{Re}(\hat{\mu})}}\left(1+i \alpha_{\mu}\right)
$$

For the sake of simplicity in this work, the material damping ratios are assumed to be the same, i.e., $\alpha_{\mu}=\alpha_{\lambda}$. The complex P-wavenumber $\hat{k}_{p}$ and the complex Poisson's ratio $\hat{\nu}$ are given by (note that the Poisson's ration thus reduce to the real Poisson's ratio):

$$
\hat{k}_{p}=\hat{k}_{s} \sqrt{\frac{\operatorname{Re}(\hat{\mu})}{\operatorname{Re}(\hat{\lambda})+2 \operatorname{Re}(\hat{\mu})}}, \quad \nu=\operatorname{Re}(\hat{\nu})=\frac{\operatorname{Re}(\hat{\lambda})}{2(\operatorname{Re}(\hat{\lambda})+\operatorname{Re}(\hat{\mu}))}
$$


It follows that $\alpha_{\mu}=\alpha_{\lambda}=\alpha_{s}$. In practice, to consider weak dissipation, values of attenuation should be contained in the range $0 \leqslant Q^{-1}(\omega) \leqslant 0.2$, which implies $0 \leqslant \alpha(\omega) \leqslant 0.1$. Damping in real soils follows in fact the weak-dissipation assumption, with typical values in the range $\alpha_{s} \in[0.03,0.06]$. Table 5 gives the compression rates $\tau$ (expressed in thoussandths for more readability) for various admissibility conditions and realistic damping ratios $\alpha_{s}$.

\begin{tabular}{|c|c|c|c|c|c|c|c|c|}
\hline Damping ratio $\alpha_{s}$ & $\begin{array}{c}\tau \text { with } k_{s}=18 \\
\text { 6] only }\end{array}$ & $\begin{array}{c}\tau \text { with } k_{s}=18 \\
6]+9 . \\
\varepsilon_{\text {decay }}=10^{-10}\end{array}$ & $\begin{array}{c}\tau \text { with } k_{s}=18 \\
10 \mathrm{~b})+10 \mathrm{c} \\
\eta_{2}=2\end{array}$ & $\begin{array}{c}\tau \text { with } k_{s}=18 \\
\begin{array}{c}10 \mathrm{~b}] \\
\eta_{2}=3\end{array}\end{array}$ & $\begin{array}{c}\tau \text { with } k_{s}=90 \\
\text { 6] only }\end{array}$ & $\begin{array}{c}\tau \text { with } k_{s}=90 \\
6]+9] \\
\varepsilon_{\text {decay }}=10^{-10}\end{array}$ & $\begin{array}{c}\tau \text { with } k_{s}=90 \\
\begin{array}{c}10 \mathrm{~b}] \\
\eta_{2}=2\end{array}\end{array}$ & $\begin{array}{c}\tau \text { with } k_{s}=90 \\
\begin{array}{c}10 \mathrm{~b}] \\
\eta_{2}=3\end{array}\end{array}$ \\
\hline 0 & 317 & 317 & 1000 & 1000 & 33.2 & 33.2 & 404 & 250 \\
\hline 0.03 & 317 & 317 & 1000 & 1000 & 32.5 & 32.5 & 310 & 160 \\
\hline 0.04 & 317 & 317 & 1000 & 1000 & 32.0 & 32.0 & 291 & 160 \\
\hline 0.05 & 317 & 317 & 1000 & 1000 & 31.4 & 31.4 & 267 & 160 \\
\hline 0.06 & 316 & 316 & 1000 & 1000 & 30.7 & 30.7 & 235 & 157 \\
\hline
\end{tabular}

Table 5: Evolution of the compression rate $\tau$ (expressed in thousandths for more readability) with respect to the damping ratio for different admissibility conditions $\left[k_{s} a=\{18,18 \times 5\}\right.$ with 10 points per $\left.\lambda_{s}, N_{\text {leaf }}=100\right]$.

We understand at this point that if some savings will be obtain in the case of viscoelastodynamic problems compared to the same purely elastic problem, it is not the most interesting domain of application of the proposed new criterion. Hopefully, this approach can be very efficient for purely elastic time-domain problems.

5.2 Efficiency of the approach in the context of the convolution Quadrature Method for 3D time-domain elastodynamics

Another interesting configuration in which purely elastodynamic problems are consider with a complex wave number is when a CQM-based approach is used to reformulate the time-domain BIE in terms of BIEs in the (complex) frequency domain. The approach can conveniently be presented by focusing on the evaluation of the single-layer integral operator $G\{f\}$ for a given causal density $f$ (see [28] for more details in the context of Helmholtz problems). It is based on a numerical approximation of convolution integrals such as:

$$
f * g(x)=\int_{0}^{x} f(x-t) g(t) d t, x \geq 0
$$

by quadrature rules. This method has been introduced in [26] where the theoretical procedure to obtain an approximation of a convolution product at discrete times (with constant time-step) is obtained and then extended in [25] with variable time-steps. [27] gives the numerical evaluation of the quadrature weights. Using the CQM, the influence of the damping in case of viscoor poroelasticity can be taken into account (see [34], [33]). The Z-BEM method refers to the whole solving procedure used here. The use of $\mathcal{Z}$-transform in the context of the CQM eases the approximation of convolution products appearing in the initial time-domain problem. The inverse $\mathcal{Z}$-transform is used to express the discrete time-domain solution obtained once the BIE is solved. This procedure is detailed in an acoustic configuration in [28].

We consider a classical elastodynamic problem in the time-domain. For a given force distribution $\mathbf{F}(\mathbf{y}, t)$ over the geometry studied, an elastodynamic state is any triplet $(\mathbf{u}, \boldsymbol{\sigma}, \mathbf{F})$ satisfying the linear elastic constitutive equation and the fundamental equation of motion

$$
\left\{\begin{array}{l}
\boldsymbol{\sigma}=\lambda(\operatorname{div} \mathbf{u}) \mathbf{1}+\mu\left(\nabla \mathbf{u}+\nabla^{T} \mathbf{u}\right) \\
\operatorname{div} \boldsymbol{\sigma}+\rho(\mathbf{F}-\ddot{\mathbf{u}})=0 \\
\mathbf{u}(\mathbf{y}, 0)=\mathbf{u}_{0}(\mathbf{y}) \dot{\mathbf{u}}(\mathbf{y}, 0)=\mathbf{v}_{0}(\mathbf{y}) \\
+ \text { prescribed boundary conditions }
\end{array} \forall(\mathbf{y}, t) \in \Omega \times[0, T]\right.
$$

From [26, we deduce the integral representation for an elastodynamic state [5]

$$
\begin{gathered}
u_{k}(\mathbf{x}, t)+\int_{\partial \Omega}\left\{T_{i}^{k}(\mathbf{x}, t, \mathbf{y}) * u_{i}(\mathbf{y}, t)-U_{i}^{k}(\mathbf{x}, t, \mathbf{y}) * t_{i}(\mathbf{y}, t)\right\} d S_{y}=\int_{\Omega} \rho U_{i}^{k}(\mathbf{x}, t, \mathbf{y}) * F_{i}(\mathbf{y}, t) d V_{y} \\
+\int_{\Omega} \rho\left\{v_{0 i}(\mathbf{y}) U_{i}^{k}(\mathbf{x}, t, \mathbf{y})+u_{0 i}(\mathbf{y}) \dot{U}_{i}^{k}(\mathbf{x}, t, \mathbf{y})\right\} d V_{y}
\end{gathered}
$$

where $T_{i}^{k}=\Sigma_{i j}^{k} n_{j}$ are the components of the traction vector associated with the fundamental solution. By applying a limiting process [5], we obtain the following regularized displacement integral equation [5]

$$
\int_{\partial \Omega}\left[T_{i}^{k}(\mathbf{x}, t, \mathbf{y}) * u_{i}(\mathbf{y}, t)-u_{i}(\mathbf{x}, t) T_{i}^{k}(\mathbf{x}, \mathbf{y})\right] d S_{y}-\int_{\partial \Omega} U_{i}^{k}(\mathbf{x}, t, \mathbf{y}) * t_{i}(\mathbf{y}, t) d S_{y}=0
$$


To present the $\mathcal{Z}$-BEM approach [28] to solve $[28]$ in the time domain we focus on the evaluation of the single-layer integral operator:

$$
q(t)=\int_{\partial \Omega} U_{i}^{k}(\mathbf{x}, t, \mathbf{y}) * t_{i}(\mathbf{y}, t) d S_{y}=\int_{\partial \Omega} \int_{0}^{t} U_{i}^{k}(\mathbf{x}, t-\tau, \mathbf{y}) * t_{i}(\mathbf{y}, \tau) d \tau d S_{y}
$$

The starting point is to note that $U_{i}^{k}(\mathbf{x}, t-\tau, \mathbf{y})$ may be expressed in terms of its Laplace transform $\bar{U}_{i}^{k}$ (assuming it is well-defined), so that for $\mathbf{x}$ and $\mathbf{y}$ fixed:

$$
q(t)=\int_{0}^{t}\left(\frac{1}{2 \pi i} \int_{\gamma-i . \infty}^{\gamma+i . \infty} \overline{U_{i}^{k}}(s) e^{s(t-\tau)} d s\right) t_{i}(\tau) d \tau=\frac{1}{2 \pi i} \int_{\gamma-i . \infty}^{\gamma+i . \infty} \overline{U_{i}^{k}}(s) h(\tau ; s) d s, \text { with } h(t ; s):=\int_{0}^{t} e^{s(t-\tau)} t_{i}(\tau) d \tau .
$$

Considering a sequence of discrete time instants $t_{n}=n \Delta t, n \in \mathbb{N}$, where $\Delta t$ is the constant time step, the CQM is developed as a means to evaluate the sequence $\left(q_{n}\right)_{n \geq 0}$ of convolution values $q_{n}:=q\left(t_{n}\right)$, over a finite discrete time interval $\{0, \Delta t, 2 \Delta t \ldots, T=M \Delta t\}$, given the sequence $\left(t_{i_{n}}\right):=\left(t_{i}\left(t_{n}\right)\right)_{n \geq 0}$ and the fundamental solution $U_{i}^{k}$. The key point of the CQM consists then in remarking that the function $t \longrightarrow h(t ; s)$ introduced in 29$)$ satisfies the initial-value problem:

$$
\left\{\begin{array}{c}
\frac{d h}{d t}(t ; s)=s h(t ; s)+t_{i}(t) \\
h(t \leqslant 0 ; s)=0
\end{array}\right.
$$

We can numerically solve the ordinary differential equation 30 for the time-discrete approximation: $h_{n}(s):=h\left(t_{n}, s\right)$ of $h(t ; s)$ (with fixed $s$ ) by applying for example a linear multi-step method to 30 ) such that

$$
\left\{\begin{array}{cl}
\frac{d h_{n}(s)}{d t} \simeq \frac{1}{\Delta t} \sum_{j=0}^{k} \alpha_{j} h_{n+j-k}(s)=\sum_{j=0}^{k} \beta_{j}\left(s h_{n+j-k}(s)+t_{i_{n+j-k}}\right), & \forall n \in \mathbb{N}, \\
h_{-p}=t_{i_{-p}}=0 & \forall p \in[|1, k|]
\end{array}\right.
$$

where the coefficients $\alpha_{j}$ and $\beta_{j}$ are the constants of the multistep method (for instance, $k=2, \alpha_{0}=1, \alpha_{1}=-4, \alpha_{2}=$ $3, \beta_{0}=\beta_{1}=0, \beta_{2}=2$ for the Backward Differentiation Formula of order 2 (BDF2) method). The $\mathcal{Z}$-transform $\mathcal{Z}\left[\left(x_{n}\right)\right](\xi)$ of a discrete-time signal $\left(x_{n}\right)$ is given for $|\xi| \leqslant \varrho$ (with $\varrho$ the radius of convergence of the series) by

$$
\mathcal{Z}:\left(x_{n}\right)=\left\{x_{0}, x_{1}, \ldots\right\} \longrightarrow \mathcal{Z}\left[\left(x_{n}\right)\right](\xi)=\sum_{n=0}^{\infty} x_{n} \xi^{n} \equiv \mathbf{X}(\xi), \xi \in \mathbb{C}
$$

Multiplying by $\Delta t \xi^{n}$, taking the $\mathcal{Z}$-transform of $(31$ ) and taking the sum over $n$, we obtain after some manipulations

$$
\sum_{j=0}^{k} \alpha_{j} \xi^{k-j} \mathbf{H}(\xi ; s)=\Delta t \sum_{j=0}^{k} \beta_{j} \xi^{k-j}\left(s \mathbf{H}(\xi ; s)+\mathbf{T}_{i}(\xi)\right)
$$

such that

$$
\mathbf{H}(\xi, s)=\frac{1}{\frac{p(\xi)}{\Delta t}-s} \mathbf{T}_{i}(\xi) \quad \text { with } \quad p(\xi)=\frac{\sum_{j=0}^{k} \alpha_{j} \xi^{k-j}}{\sum_{j=0}^{k} \beta_{j} \xi^{k-j}}
$$

$p$ is characteristic of the multistep method chosen: $p(\xi)=\left(3-4 \xi+\xi^{2}\right) / 2$ for the BDF2 method and $p(\xi)=\left(11-18 \xi+9 \xi^{2}-\right.$ $\left.\xi^{3}\right) / 6$ for the BDF3 method. With the help of the Cauchy's residue theorem, we obtain an approximation of the $\mathcal{Z}$-transform of the convolution product

$$
\mathbf{Q}(\xi)=\overline{\mathbf{U}}_{i}^{k}\left(\frac{p(\xi)}{\Delta t}\right) \mathbf{T}_{i}(\xi) .
$$

We can then extend this result to the whole BIE (28). It involves the numerical resolution of distinct BIEs in the complex frequency domain given by the discrete values of $s: s_{p}=p\left(\xi_{p}\right) / \Delta t$ with $\xi_{p}=\rho e^{2 i \pi p / L}, L$ complex numbers taken on the circle of radius $\rho$ in the complex space. Once the solution $\mathbf{U}_{i}(., \xi)$ is obtained, the time discrete physical unknowns $u_{i}\left(., t_{n}\right)$ are obtained by taking the inverse $\mathcal{Z}$-transform of $\mathbf{U}_{i}(., \xi)$ given by:

$$
u_{i}\left(., t_{n}\right)=\frac{1}{2 i \pi} \int_{C} \mathbf{U}_{i}(., \xi) \xi^{-k-1} d \xi \simeq \frac{1}{L} \sum_{p=0}^{L-1} \mathbf{U}_{i}\left(., \xi_{p}\right) \xi_{p}^{-k}, \quad \forall k \in[|0, M|]
$$

with $M$ the total number of time steps. Figure 15 illustrates the complex frequencies $s_{p}$ at which the solution of the BIE is required for $\Delta t=10^{-4}$ and for the BDF2 (Fig. 15a) and BDF3 (Fig. 15b) linear multistep methods in 31). Figure 15 illustrates that the $\mathcal{Z}$-BEM implies the solutions of BEM problems with complex wavenumbers for which the decay ratio $\alpha_{s}=\operatorname{Im}\left(k_{s}\right) / \operatorname{Re}\left(k_{s}\right)$ is taken in a large range.

For our examples with $\Delta t=10^{-4}, \min \alpha_{s}=0, \max \alpha_{s}=215$ for BDF2 and $\max \alpha_{s}=16240$ for BDF3. Due to the definition of $s_{p}$, these numbers will increase if $\Delta t$ is decreased. For each integration contour in Figure 15, Table 6 gives 


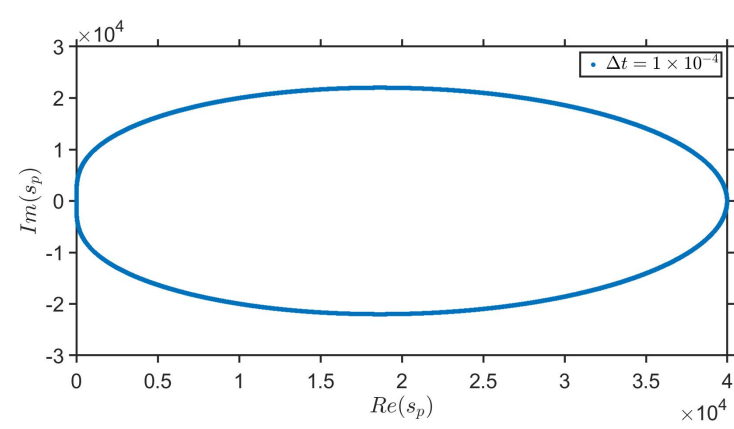

(a) $s_{p}$ values for the BDF2 linear multistep method

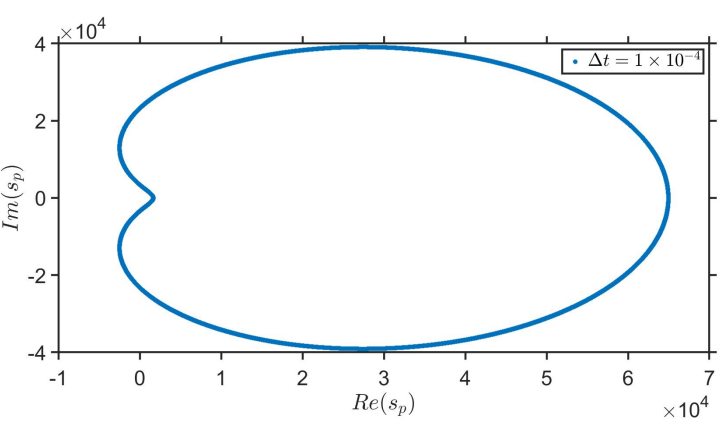

(b) $s_{p}$ values for the BDF3 linear multistep method

Fig. 15: Complex frequencies $s_{p}$ for which BEM problems have to be solved in the $\mathcal{Z}$-BEM for two different linear multistep methods and a time step $\Delta t=10^{-4}$.

the number of complex frequencies lying inside some damping ratio intervals. It illustrates the repartition of the interesting damping ratios for this application. Finally, Tables 7 and 8 give the compression rates $\tau$ (expressed in thousandths for more readability) for various admissibility conditions and the damping ratios $\alpha_{s}$ observed in Figures $15 \mathrm{a}$ and $15 \mathrm{~b}$ respectively, with the help of Table 6. As expected, we observe that the compression increases with the frequency. Another interesting behaviour is that the standard admissibility condition (6) produces similar results than $(10 \mathrm{~b})+(10 \mathrm{c})$. Importantly, when the damping ratio increases, we observe that the obtained compression reaches a plateau. Thus the introduction of more damping does not change the compression rate. On the other hand, the proposed approach with $(6)+(9)$ enables an increase of the gain as the damping ratio increases. This means that the additional compression is obtained by approximating the non-admissible blocks. For the largest damping ratios, we notice that the proposed approach enables to divide by a factor up to three the storage requirements. These results confirm the interest of the proposed approach in the context of $\mathcal{Z}$-BEM.

\begin{tabular}{ccc}
\hline Intervals of $\alpha_{s}$ & $\begin{array}{c}\text { Number of complex frequencies } \\
\text { for BDF2 integration contour } \\
\text { Figure } 15 \mathrm{a}\end{array}$ & $\begin{array}{c}\text { Number of complex frequencies } \\
\text { for BDF3 integration contour } \\
\text { Figure } 15 \mathrm{~b}\end{array}$ \\
\hline$[0,1]$ & 6850 & 6580 \\
{$[1,10]$} & 8080 & 10702 \\
{$[10,30]$} & 1830 & 1826 \\
{$[30,60]$} & 898 & 448 \\
{$[60,100]$} & 646 & 178 \\
{$[100,600]$} & 1696 & 222 \\
{$[600,17000]$} & 0 & 44 \\
\hline
\end{tabular}

Table 6: Number of complex frequencies lying in some damping ratio intervals for BDF2 and BDF3 integration contours.

\begin{tabular}{|c|c|c|c|c|c|c|c|c|}
\hline Damping ratio $\alpha_{s}$ & $\begin{array}{c}\tau \text { with } k_{s}=18 \\
\text { [6] only }\end{array}$ & $\begin{array}{c}\tau \text { with } k_{s}=18 \\
6]+9 \\
\varepsilon_{\text {decay }}=10^{-10}\end{array}$ & $\begin{array}{c}\tau \text { with } k_{s}=18 \\
10 \mathrm{~b}+10 \mathrm{c} \\
\eta_{2}=2\end{array}$ & $\begin{array}{c}\tau \text { with } k_{s}=18 \\
10 \mathrm{~b}+10 \mathrm{c} \\
\eta_{2}=3\end{array}$ & $\begin{array}{c}\tau \text { with } k_{s}=90 \\
\text { 6] only }\end{array}$ & $\begin{array}{c}\tau \text { with } k_{s}=90 \\
6]+9] \\
\varepsilon_{\text {decay }}=10^{-10}\end{array}$ & $\begin{array}{c}\tau \text { with } k_{s}=90 \\
10 \mathrm{~b}+10 \mathrm{c} \\
\eta_{2}=2\end{array}$ & $\begin{array}{c}\tau \text { with } k_{s}=90 \\
\begin{array}{c}10 \mathrm{~b}) \\
\eta_{2}=3\end{array}\end{array}$ \\
\hline 0 & 317 & 317 & 345 & 345 & 33.2 & 33.2 & 404 & 250 \\
\hline 5 & 205 & 205 & 278 & 254 & 6.57 & 6.57 & 11.0 & 11.0 \\
\hline 10 & 202 & 202 & 278 & 209 & 6.17 & 6.17 & 8.67 & 7.87 \\
\hline 20 & 202 & 141 & 278 & 209 & 6.16 & 4.73 & 8.67 & 6.44 \\
\hline 30 & 202 & 130 & 278 & 209 & 6.16 & 4.44 & 8.67 & 6.44 \\
\hline 40 & 202 & 119 & 278 & 209 & 6.16 & 4.22 & 8.67 & 6.44 \\
\hline 50 & 202 & 117 & 278 & 209 & 6.16 & 4.07 & 8.67 & 6.44 \\
\hline 100 & 202 & 104 & 278 & 209 & 6.16 & 3.54 & 8.67 & 6.44 \\
\hline 130 & 202 & 103 & 278 & 209 & 6.16 & 3.42 & 8.67 & 6.44 \\
\hline 160 & 202 & 103 & 278 & 209 & 6.16 & 3.33 & 8.67 & 6.44 \\
\hline 200 & 202 & 102 & 278 & 209 & 6.16 & 3.25 & 8.67 & 6.44 \\
\hline 215 & 202 & 102 & 278 & 209 & 6.16 & 3.22 & 8.67 & 6.44 \\
\hline
\end{tabular}

Table 7: Evolution of the compression rate $\tau$ (expressed in thousandths for more readability) with respect to reachable damping ratios in BDF2 integration's contour Figure $15 \mathrm{a}$ for different admissibility conditions $\left[k_{s} a=\{18,90\}\right.$, with 10 points per $\left.\lambda_{s}, N_{\text {leaf }}=100\right]$. 


\begin{tabular}{|c|c|c|c|c|c|c|c|c|}
\hline Damping ratio $\alpha_{s}$ & $\begin{array}{c}\tau \text { with } k_{s}=18 \\
\text { 6] only }\end{array}$ & $\begin{array}{c}\tau \text { with } k_{s}=18 \\
6+9 \\
\varepsilon_{\text {decay }}=10^{-10}\end{array}$ & $\begin{array}{c}\tau \text { with } k_{s}=18 \\
\begin{array}{c}10 \mathrm{~b}] \\
\eta_{2}=2\end{array}\end{array}$ & $\begin{array}{c}\tau \text { with } k_{s}=18 \\
10 \mathrm{~b}+10 \mathrm{c} \\
\eta_{2}=3\end{array}$ & $\begin{array}{c}\tau \text { with } k_{s}=90 \\
6 \text { only }\end{array}$ & $\begin{array}{c}\tau \text { with } k_{s}=90 \\
6+9 \\
\varepsilon_{\text {decay }}=10^{-10}\end{array}$ & $\begin{array}{c}\tau \text { with } k_{s}=90 \\
10 \mathrm{~b}+10 \mathrm{c} \\
\eta_{2}=2\end{array}$ & $\begin{array}{c}\tau \text { with } k_{s}=90 \\
10 \mathrm{~b}+10 \mathrm{c} \\
\eta_{2}=3\end{array}$ \\
\hline 0 & 317 & 317 & 345 & 345 & 33.2 & 33.2 & 404 & 250 \\
\hline 5 & 205 & 205 & 278 & 254 & 6.57 & 6.57 & 11.0 & 11.0 \\
\hline 10 & 202 & 202 & 278 & 209 & 6.17 & 6.17 & 8.67 & 7.87 \\
\hline 20 & 202 & 141 & 278 & 209 & 6.16 & 4.73 & 8.67 & 6.44 \\
\hline 30 & 202 & 130 & 278 & 209 & 6.16 & 4.44 & 8.67 & 6.44 \\
\hline 40 & 202 & 119 & 278 & 209 & 6.16 & 4.22 & 8.67 & 6.44 \\
\hline 50 & 202 & 117 & 278 & 209 & 6.16 & 4.07 & 8.67 & 6.44 \\
\hline 100 & 202 & 104 & 278 & 209 & 6.16 & 3.54 & 8.67 & 6.44 \\
\hline 130 & 202 & 103 & 278 & 209 & 6.16 & 3.42 & 8.67 & 6.44 \\
\hline 160 & 202 & 103 & 278 & 209 & 6.16 & 3.33 & 8.67 & 6.44 \\
\hline 200 & 202 & 102 & 278 & 209 & 6.16 & 3.25 & 8.67 & 6.44 \\
\hline 500 & 202 & 99.5 & 278 & 209 & 6.16 & 3.06 & 8.67 & 6.44 \\
\hline 1000 & 202 & 97.5 & 278 & 209 & 6.16 & 2.92 & 8.67 & 6.44 \\
\hline 10000 & 202 & 94.9 & 278 & 209 & 6.16 & 2.78 & 8.67 & 6.44 \\
\hline 16240 & 202 & 94.9 & 278 & 209 & 6.16 & 2.78 & 8.67 & 6.44 \\
\hline
\end{tabular}

Table 8: Evolution of the compression rate $\tau$ (expressed in thousandths for more readability) with respect to reachable damping ratios in BDF3 integration's contour Figure $15 \mathrm{~b}$ for different admissibility conditions $\left[k_{s} a=\{18,90\}\right.$ with 10 points per $\left.\lambda_{s} N_{\text {leaf }}=100\right]$.

\section{Conclusion}

In this work, we have evolved the admissibility condition in the $\mathcal{H}$-BEM to simulate $3 \mathrm{D}$ elastodynamic problems with complex wavenumbers. This evolution, which consists in adding condition (9) to the classical condition (6), is a way to obtain improved data-sparse approximations of the discretized Green's tensor. It takes into account the influence of the imaginary part of the complex wavenumbers on the exponential decay induced in the Green's tensor. This exponential decay has a moderate influence on the data-sparse approximation of the matrix in the viscoelastodynamic regime. However it has a much stronger influence on the data sparse approximation of the matrix in the context of the convolution quadrature method for BEMs 3D elastodynamics. The literature on this topic is so far quite limited. In [12] a new admissibility condition to study highly-oscillatory Helmholtz kernels with complex wave numbers in the context of directional $\mathcal{H}^{2}$-matrices is proposed. We have shown that our new admissibility condition is simpler to implement and allows one to achieve improved compression rates with a controlled loss of accuracy. We use the sound theoretical background proposed in [12] to back up the efficiency of our approach.

It is the authors' belief that the present study could have a real impact on the efficiency of the $\mathcal{Z}$-BEM approach for 3D elastodynamics (and Helmholtz) in the time domain. Among others, we have shown that the gain in terms of memory requirements becomes significant when the damping ratio is above a threshold value. In such configurations, the storage requirement converges towards a minimal storage representative of non-admissible blocks only. Indeed, the non-admissible blocks have a fixed position in the matrix, and the discretized Green's tensor can be accurately approximated by these nonadmissible blocks only, as the strong exponential decay allows one to approximate all the admissible part of the matrix by matrices of zeros. Figure 16 summarises the range of values reached by $\alpha_{s}$ when the $\mathcal{Z}$-BEM approach is used for purely elastic problems and for viscoelastodynamic problems.

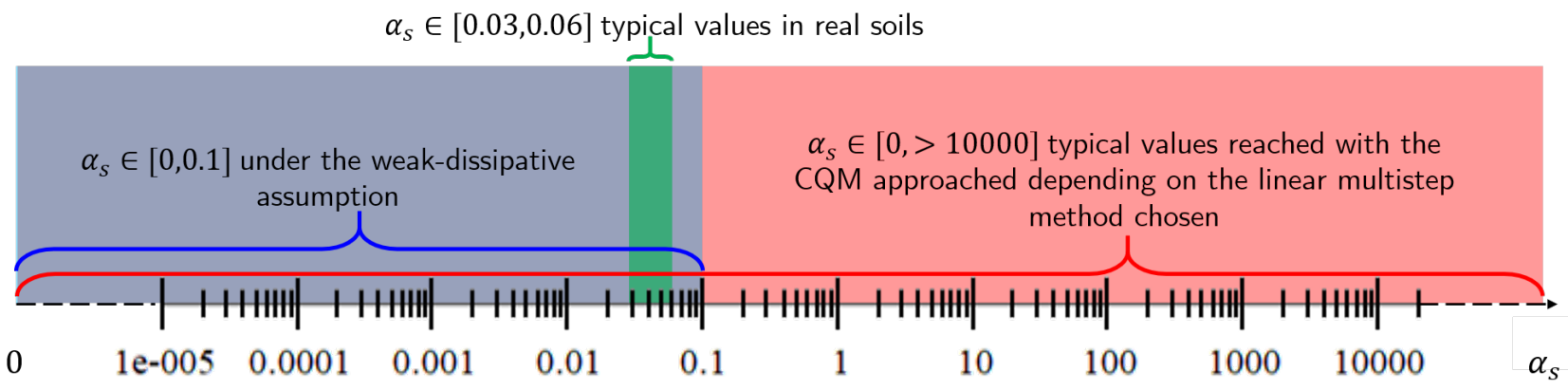

Fig. 16: Values taken by $\alpha_{s}$ in case of the $\mathcal{Z}$-BEM approach for time-domain elastodynamics and for viscoelastodynamic problems. 


\section{References}

1. L. Banjai and W. Hackbusch. Hierarchical matrix techniques for low-and high-frequency Helmholtz problems. IMA journal of numerical analysis, 28(1):46-79, 2008.

2. M. Bebendorf. Hierarchical matrices. Springer, 2008.

3. M. Bebendorf, C. Kuske, and R. Venn. Wideband nested cross approximation for Helmholtz problems. Numerische Mathematik, $130(1): 1-34,2015$.

4. M. Bebendorf and S. Rjasanow. Adaptive low-rank approximation of collocation matrices. Computing, 70(1):1-24, 2003.

5. M. Bonnet. Boundary integral equations methods in solids and fluids. John Wiley and sons, 1999.

6. S. Börm. Data-sparse approximation of non-local operators by h2-matrices. Linear algebra and its applications, 422(2-3):380-403, 2007.

7. S. Börm. Directional-matrix compression for high-frequency problems. Numerical Linear Algebra with Applications, $24(6):$ 2112, 2017.

8. S. Börm and S. Christophersen. Approximation of integral operators by Green quadrature and nested cross approximation. Numerische Mathematik, 133(3):409-442, 2016.

9. S. Börm, L. Grasedyck, and W. Hackbusch. Introduction to hierarchical matrices with applications. Engineering Analysis with Boundary Elements, 27(5):405-422, 2003.

10. S. Börm. Fast large-scale boundary element algorithms. arXiv:2001.05523 [cs, math], June 2020. arXiv: 2001.05523.

11. S. Börm and C. Börst. Hybrid Matrix Compression for High-Frequency Problems. SIAM Journal on Matrix Analysis and Applications, 41(4):17041725, January 2020. Publisher: Society for Industrial and Applied Mathematics.

12. S. Börm, M. Lopez-Fernandez, and S.A. Sauter. Variable Order, Directional $\mathcal{H}^{2}$-Matrices for Helmholtz Problems with Complex Frequency. IMA Journal of Numerical Analysis, 12 2020. draa046.

13. S. Chaillat and M. Bonnet. Recent advances on the fast multipole accelerated boundary element method for $3 \mathrm{~d}$ time-harmonic elastodynamics. Wave Motion, 50(7):1090-1104, 2013.

14. S. Chaillat, L. Desiderio, and P. Ciarlet. Theory and implementation of H-matrix based iterative and direct solvers for Helmholtz and elastodynamic oscillatory kernels. Journal of Computational Physics, 351:165-186, December 2017.

15. E. Darve. The fast multipole method: numerical implementation. Journal of Computational Physics, 160(1):195-240, 2000.

16. G.H. Golub and C.F. Van Loan. Matrix computations. JHU Press, 3rd edition, 2012.

17. E. Grasso. Modelling visco-elastic seismic wave propagation : a fast-multipole boundary element method and its coupling with finite elements. phdthesis, Université Paris-Est, June 2012.

18. E. Grasso, S. Chaillat, M. Bonnet, and J.F. Semblat. Application of the multi-level time-harmonic fast multipole BEM to 3-D visco-elastodynamics. Engineering Analysis with Boundary Elements, 36(5):744-758, May 2012.

19. L. Greengard, J. Huang, V. Rokhlin, and S. Wandzura. Accelerating fast multipole methods for the helmholtz equation at low frequencies. IEEE Computational Science and Engineering, 5(3):32-38, 1998.

20. L. Greengard and V. Rokhlin. A fast algorithm for particle simulations. Journal of computational physics, 73(2):325-348, 1987.

21. W. Hackbusch. A sparse matrix arithmetic based on $\mathcal{H}$-matrices. Part I: Introduction to $\mathcal{H}$-matrices. Computing, 62(2):89-108, 1999.

22. W. Hackbusch. Hierarchical matrices: algorithms and analysis, volume 49. Springer, 2015.

23. W. Hackbusch and Z.P. Nowak. On the fast matrix multiplication in the boundary element method by panel clustering. Numerische Mathematik, 54(4):463-491, 1989.

24. Y.J. Liu, S. Mukherjee, N. Nishimura, M. Schanz, W. Ye, A. Sutradhar, E. Pan, N.A. Dumont, A. Frangi, and A. Saez. Recent advances and emerging applications of the boundary element method. Applied Mechanics Reviews, 64(3), 2011.

25. M. Lopez-Fernandez and S. Sauter. Generalized convolution quadrature with variable time stepping. IMA Journal of Numerical Analysis, 33(4):11561175, October 2013. Conference Name: IMA Journal of Numerical Analysis.

26. C. Lubich. Convolution quadrature and discretized operational calculus. I. Numerische Mathematik, 52(2):129-145, January 1988.

27. C. Lubich. Convolution quadrature and discretized operational calculus. II. Numerische Mathematik, 52(4):413-425, July 1988.

28. D. Mavaleix-Marchessoux, M. Bonnet, S. Chaillat, and B. Leblé. A fast BEM procedure using the Z-transform and high-frequency approximations for large-scale 3D transient wave problems. International Journal for Numerical Methods in Engineering, 2020. Publisher: Wiley.

29. M. Messner, M. Schanz, and E. Darve. Fast directional multilevel summation for oscillatory kernels based on Chebyshev interpolation. Journal of Computational Physics, 231(4):1175-1196, 2012.

30. V. Rokhlin. Rapid solution of integral equations of classical potential theory. Journal of computational physics, 60(2):187-207, 1985.

31. S. Sauter. Variable order panel clustering. Computing, 64(3):223-261, 2000.

32. S.A. Sauter and C. Schwab. Boundary element methods. In Boundary Element Methods, pages 183-287. Springer, 2010.

33. M. Schanz. Application of 3D time domain boundary element formulation to wave propagation in poroelastic solids. Engineering Analysis with Boundary Elements, 25(4):363-376, April 2001.

34. M. Schanz and H. Antes. A new visco- and elastodynamic time domain Boundary Element formulation. Computational Mechanics, 20(5):452-459, October 1997.

35. J.-F. Semblat and M. P. Luong. Wave propagation through soils in centrifuge testing. Journal of Earthquake Engineering, 02(01):147-171, January 1998. Publisher: Imperial College Press.

36. D.R. Wilkes and A.J. Duncan. A low frequency elastodynamic fast multipole boundary element method in three dimensions. Computational Mechanics, 56(5):829-848, 2015. 